\title{
Long-range vector models at large $\mathbf{N}$
}

\section{Noam Chai, Mikhail Goykhman and Ritam Sinha}

The Racah Institute of Physics, The Hebrew University of Jerusalem, Jerusalem 91904, Israel

E-mail: noam.chai@mail.huji.ac.il, michael.goykhman@mail.huji.ac.il, ritam.sinha@mail.huji.ac.il

ABSTRACT: We calculate various CFT data for the $O(N)$ vector model with the long-range interaction, working at the next-to-leading order in the $1 / N$ expansion. Our results provide additional evidence for the existence of conformal symmetry at the long-range fixed point, as well as the continuity of the CFT data at the long-range to short-range crossover point $s_{\star}$ of the exponent parameter $s$. We also develop the $N>1$ generalization of the recently proposed IR duality between the long-range and the deformed short-range models, providing further evidence for its non-perturbative validity in the entire region $d / 2<s<s_{\star}$.

KEywords: 1/ $N$ Expansion, Conformal Field Theory, Field Theories in Lower Dimensions, Nonperturbative Effects

ARXIV EPRINT: 2107.08052 


\section{Contents}

1 Introduction 1

2 Set-up 5

3 Two-point functions $\quad 8$

$3.1\langle\phi \phi\rangle \quad 9$

$3.2\langle\sigma \sigma\rangle \quad 9$

4 Conformal invariance and composite operators 12

$4.1\left\langle\sigma^{2} \sigma^{2}\right\rangle \quad 12$

$\begin{array}{ll}4.2\left\langle\sigma^{n} \sigma^{n}\right\rangle & 15\end{array}$

$\begin{array}{ll}4.3 \text { The }\left\langle\sigma^{2} \sigma\right\rangle \text { correlator and conformal invariance } & 15\end{array}$

$4.4\langle\sigma \phi \sigma \phi\rangle) 17$

$\begin{array}{llr}5 & \text { OPE coefficients } & 19\end{array}$

$\begin{array}{lll}5.1\langle\phi \phi \sigma\rangle & 19\end{array}$

$5.2\langle\sigma \sigma \sigma\rangle \quad 24$

6 Continuity of CFT data across long-range-short-range crossover $\quad 25$

6.1 Continuity of dimensions of $\sigma, \sigma^{2}$, and $\sigma \phi \quad 25$

6.2 Continuity of $\langle\phi \phi \sigma\rangle$ and $\langle\sigma \sigma \sigma\rangle \quad 27$

7 A dual description for the long-range CFT at large $N \quad 28$

7.1 Anomalous dimension of the stress-energy tensor 32

8 Discussion $\quad 35$

$\begin{array}{ll}\text { A Some useful identities } & 36\end{array}$

\section{Introduction}

Long-range spin models provide a simple generalization of the usual Ising spin chain with the nearest neighbor interactions. However, they are known to display a much richer phase structure. The long-range Ising (LRI) model was introduced by Dyson more than fifty years ago to describe spontaneous symmetry breaking and long-range order in a $1 d$ ferromagnetic spin chain [1]. It features a spin-spin interaction that decays as a power law, controlled by a positive-valued exponent $s$. In a general dimension $d$, the Hamiltonian for the LRI model is given by

$$
H=-J \sum_{i, j} \frac{s_{i} s_{j}}{|i-j|^{d+s}}
$$


where $J>0$ governs the strength of interaction and $s_{i}= \pm 1$ are the Ising spins. This model has been subjected to numerous tests, both analytical and numerical, and was found to exhibit a second-order phase transition in low-dimensional spin chains [2-4]. The corresponding long-range critical regime was found in the window $d / 2<s<s_{\star}$ for the parameter $s$. For the range $s \leq d / 2$, the model is described by a Gaussian mean-field theory (MFT), whereas for $s \geq s_{\star}$, the model transitions to its short-range counterpart, given by the usual Ising CFT.

It is easy to identify these phases in the parameter space of $s$ in the continuum description of (1.1), obtained using the Landau-Ginzburg technique,

$$
S \propto-\int d^{d} x \int d^{d} y \frac{\phi(x) \phi(y)}{|x-y|^{d+s}} .
$$

Using (1.2), the scaling dimension of the field $\phi$ is found to be $\Delta_{\phi}=(d-s) / 2$. It follows that we need $s \leq 2$, in order for the long-range model to be unitary. One can subsequently deform the above action by adding to it a usual local quartic interaction term [2],

$$
S \propto-\int d^{d} x \int d^{d} y \frac{\phi(x) \phi(y)}{|x-y|^{d+s}}+g \int d^{d} x \phi(x)^{4} .
$$

When $s \leq d / 2$, the quartic interaction is irrelevant, and the model is described by the Gaussian MFT. When $s>d / 2$, the quartic interaction becomes relevant, and triggers an RG flow, taking the theory away from the Gaussian fixed point. One can perform a systematic perturbative expansion near $s=d / 2$ to identify a non-trivial interacting IR fixed point of this flow. This fixed point has been studied extensively in the literature (see [5-9] for a rigorous renormalization group derivation of this fixed point), and much is known about the corresponding critical exponents. The scaling dimension of the operator $\phi$ at this long-range fixed point is exact, being protected by the bi-local kinetic term in (1.3) from receiving anomalous contributions. This fixed point is also characterized by the lack of a local stress-energy tensor. The latter fact makes it rather difficult to ascertain whether the long-range fixed point in fact enjoys the full conformal symmetry. Strong support in favor of existence of conformal symmetry at the long-range fixed point has recently been provided in [10-13].

As we reviewed above, the long-range critical regime is found at the end of an RG flow in (1.3) for the exponent parameter $s$ taking values in the range $d / 2<s<s_{\star}$. The model crosses over to the short-range CFT regime when $s \geq s_{\star} \cdot{ }^{1}$ While the dimension of $\phi$ in the long range CFT is $(d-s) / 2$, its corresponding dimension in the short-range CFT is $d / 2-1+\gamma_{\hat{\phi}}$, with $\gamma_{\hat{\phi}}$ being the anomalous dimension of the short-range field $\hat{\phi}$ (in this paper we will typically put a hat on top of the letter standing for the short-range field, whenever the same letter without a hat is used to denote the long-range field). Continuity at $s_{\star}$ then implies that $s_{\star}=2-2 \gamma_{\hat{\phi}}[14,15]$. Notice that since $\gamma_{\hat{\phi}}>0$ in the usual short-range CFT, then $s_{\star}<2$, implying that interactions tighten the upper bound on $s$. In fact, it follows that all CFT data, not just the conformal dimension of $\phi$, are continuous across the

\footnotetext{
${ }^{1}$ The short-range CFT at $s>s_{\star}$ is the critical short-range Ising model plus a decoupled sector consisting of a generalized free field $[12,13]$, as we review below.
} 
crossover point $s_{\star}[14,15]$. In this paper we will illustrate such continuity by carrying out explicit calculations of CFT data in the $O(N)$ generalization of the model (1.3), working at the next-to-leading order in $1 / N$ expansion.

We begin by considering an $O(N)$ version of the non-local MFT, and perturb it by a quartic interaction. To access the strongly-interacting IR regime of this model away from the weakly-coupled behavior near $s=d / 2$, we study it in the $1 / N$ expansion. By employing the Hubbard-Stratonovich formalism we can write down the corresponding action of the model as

$$
S \propto-\int d^{d} x \int d^{d} y \frac{\phi_{i}(x) \phi_{i}(y)}{|x-y|^{d+s}}+\int d^{d} x\left(-\frac{1}{4 g} \sigma^{2}+\frac{1}{\sqrt{N}} \sigma \phi^{2}\right),
$$

where $\sigma$ is the Hubbard-Stratonovich field. The model (1.4) has been studied previously in the literature, where critical exponents such as the scaling dimensions of $\phi$ and $\sigma$ have been calculated [16-18]. However, no other CFT data in the $1 / N$ expansion is available for the long-range fixed point. One of the goals of this paper is to fill this gap, by calculating some large $N$ CFT data, including anomalous dimensions of composite operators, and various OPE coefficients. We perform most of our calculations at the next-to-leading order in the $1 / N$ expansion. Our results lend a strong support to the statement that the long-range fixed point enjoys the full conformal symmetry, even for the values of $s$ that are beyond the scope of a perturbative regime in the vicinity of $s=d / 2$.

Following our calculations of CFT data in the long-range critical $O(N)$ vector model, we proceed to establish explicitly that the obtained anomalous dimensions and OPE coefficients are continuous at $s=s_{\star}$. In fact, at $s=s_{\star}$ the long-range critical vector model crosses over to the short-range critical vector model plus a generalized free field $\chi[11,12]$. The role of the decoupled field $\chi$ becomes important when one matches the complete spectrum across the crossover point $s_{\star}$. As it was pointed out in [11, 12], some operators in the long-range model, such as $\phi^{3}$, exhibit an apparently discontinuous behavior at $s_{\star}$, having no counterpart in the spectrum of the short-range critical vector model. Introducing the generalized free field $\chi$ allows one to construct such operators when $s>s_{\star}$, thereby allowing for the complete match of the spectrum. Note that there has been some debate regarding the smooth transition of CFT data across this long-range-short-range crossover point $[19,20]$. However, our calculation provides complementary support to the numerous theoretical calculations and Monte-Carlo simulations which predicted such a smooth transition at the long-range-short-range crossover point [21-23]. ${ }^{2}$

While the long-range CFT exists in the perturbative regime near the lower bound $s=d / 2$ of the long-range window $d / 2<s<s_{\star}$, making it amenable to the Wilson-Fisher kind of $\epsilon$-expansion for $s=(d+\epsilon) / 2$, its behavior near the upper bound $s=s_{\star}$ is stronglyinteracting. It was proposed in [15] that the long-range CFT near $s=s_{\star}$ can be accessed by perturbing the short-range CFT with a bi-local kinetic term for the field $\hat{\phi}$. Such a kinetic term crosses over from being irrelevant when $s>s_{\star}$, to being relevant when $s<s_{\star}$.

\footnotetext{
${ }^{2}$ In this paper, whenever we refer to the long-range-short-range crossover point $s_{\star}$, we mean the point of crossover between the long-range critical vector model, and the short-range critical vector model with the decoupled generalized free field.
} 
Consequently, as $s$ is lowered past $s_{\star}$, an RG flow brings the model to a long-range regime in the IR. While such a bi-local kinetic term appears to manifest a perturbative behavior in the vicinity of $s_{\star}$, it is unclear how to carry out this perturbative expansion. This is primarily due to a lack of proper understanding of how to perform conformal perturbation theory involving non-local perturbations.

Recently a new weakly-coupled description near the long-range-short-range crossover point $s_{\star}$ was proposed in $[11,12]$. In this description the non-local perturbation of [15] has been traded for a local one, at the cost of introducing a generalized free field $\chi$ with scaling dimension $\Delta_{\chi}=(d+s) / 2$ into the model. The corresponding action is given by $[11,12]$

$$
S=S_{\mathrm{CFT}}+\lambda \int d^{d} x \hat{\phi} \chi+\int d^{d} x \int d^{d} y \frac{\chi(x) \chi(y)}{|x-y|^{d-s}}
$$

where $\hat{\phi}$ is the spin field and $S_{\mathrm{CFT}}$ is the action of the original short-range CFT model, and $\chi$ is a generalized free field coupled to it. The interacting term $\hat{\phi} \chi$ with the coupling $\lambda$ has the leading order scaling dimension $d-\delta$, where $\delta=\left(s_{\star}-s\right) / 2$. This perturbation is therefore slightly relevant for small positive $\delta$, that is, for $s$ slightly below the longrange-short-range crossover point $s_{\star}$. The flow triggered by this perturbation ends at a long-range fixed point in the IR, near $s=s_{\star}$. Although two different UV descriptions (1.3) and (1.5) flow to the long-range fixed points in two non-overlapping perturbative regimes, near $s=d / 2$ and $s=s_{\star}$ respectively, it has been argued in $[11,12]$ that these are in fact the same long-range fixed points for all $d / 2<s<s_{\star}$. This furnishes a non-trivial example of an IR duality. As part of matching the spectra of these two models, the new d.o.f. $\chi$ on the deformed short-range side of the duality has been suggested to be dual to the operator $\phi^{3}$ in the long-range CFT $[11,12]$.

One can further take advantage of the perturbative behavior of the coupling $\lambda$ near $s=s_{\star}$ to find the anomalous dimension of the stress-energy tensor $T_{\mu \nu}$ within conformal perturbation theory. Importantly, a non-trivial anomalous dimension of $T_{\mu \nu}$ supplies further evidence that the fixed point at the end of an RG flow triggered by the coupling $\lambda$ is indeed a long-range CFT. Additional evidence for the proposed IR duality is given by matching the ratios of the three-point function amplitudes (OPE coefficients) $\left\langle\phi \mathcal{O}_{1} \mathcal{O}_{2}\right\rangle$, $\left\langle\phi^{3} \mathcal{O}_{1} \mathcal{O}_{2}\right\rangle$ in the long-range model, and $\left\langle\hat{\phi} \mathcal{O}_{1} \mathcal{O}_{2}\right\rangle,\left\langle\chi \mathcal{O}_{1} \mathcal{O}_{2}\right\rangle$ in the deformed short-range model, for arbitrary operators $\mathcal{O}_{1,2}[11,12]$.

Inspired by the construction of $[11,12]$, we propose that the long-range $O(N)$ critical vector model for $d / 2<s<s_{\star}$ admits a dual description in terms of a deformed short-range CFT with the action

$$
S=a \int d^{d} x \int d^{d} y \frac{\chi^{i}(x) \chi^{i}(y)}{|x-y|^{d-s}}+\frac{1}{\sqrt{N}} \int d^{d} x \Sigma \Phi^{2}+\int d^{d} x \Phi^{i} \chi^{i},
$$

where $\Phi^{i}, i=1, \ldots, N$ is the spin field, $\Sigma$ is the Hubbard-Stratonovich field, and $\chi^{i}$, $i=1, \ldots, N$ is a generalized free vector field. ${ }^{3}$ We will argue that the models (1.6) and (1.4)

\footnotetext{
${ }^{3}$ The coefficient $a$ reflects a particular choice of conventions regarding normalization of $\chi^{i}$, and will be fixed below.
} 
are equivalent, up to an identification of the d.o.f.

$$
\Phi \leftrightarrow \phi, \quad \Sigma \leftrightarrow \sigma
$$

These two models, therefore, provide dual descriptions of the same long-range IR critical regime, for all values of $d / 2<s<s_{\star}$.

It then follows that the composite operator $\sigma \phi$ is dual to the field $\chi$ on the other side of the duality. While this can be seen as a direct consequence of the relations (1.7) and the e.o.m., one can additionally carry out a consistency check of the proposed duality, by matching the amplitudes of the three-point functions involving the operators $\phi, \sigma \phi$ in the

long-range model, and $\hat{\phi}, \chi[11,12]$. As we move to $s>s_{\star}$, beyond the scope of the longrange critical regime, the field $\chi$ decouples into a separate generalized free field sector. Its existence however guarantees continuity of spectrum across the crossover point $s_{\star}[11,12]$.

Following $[11,12]$, we further use our newly proposed dual IR long-range CFT description to calculate the anomalous dimension and trace of the stress-energy tensor. This calculation additionally reveals that near $s=s_{\star}$ our dual long-range CFT is perturbatively in $1 / N$ close to the short-range model in the UV, by relating anomalous dimension of the stress-energy tensor to the anomalous dimension of the spin field $\phi$.

This paper is organized as follows. In section 2, we define the action for the long-range critical $O(N)$ vector model at large $N$, and set up our conventions that will subsequently be used throughout the rest of the paper. In section 3, we compute the anomalous dimensions and amplitude corrections of the lowest scalar primaries $\phi$ and $\sigma$, up to the next-to-leading order in $1 / N$ expansion. In section 4 , we begin by calculating the anomalous dimensions of composite scalar primaries $\sigma^{n}$, at the next-to-leading order in $1 / N$, followed by the calculation of the cross-correlator $\left\langle\sigma \sigma^{2}\right\rangle$. We establish that such a cross-correlator in fact vanishes, unlike its short-range counterpart. In particular, this calculation provides additional evidence that the long-range fixed point enjoys the full conformal symmetry. In section 5, we calculate the OPE coefficients $C_{\phi \phi \sigma}$ and $C_{\sigma \sigma \sigma}$, and the corresponding conformal triangles, working at the next-to-leading order in $1 / N$. In section 6 , we explicitly demonstrate the continuity of all the calculated long-range CFT data at the long-rangeshort-range crossover point $s_{\star}$. In section 7 , we study a short-range critical $O(N)$ vector model coupled to a generalized free field $\chi^{i}$, which we propose to be dual to the long-range critical vector model in the IR. We discuss our results and outline some future research directions in section 8 .

\section{Set-up}

In this section we review the basic setup of the long-range critical $O(N)$ vector model that we will be studying in this paper. This model describes dynamics of a multiplet of $N$ scalar fields $\phi_{i}, i=1, \ldots, N$ in the fundamental representation of the $O(N)$ symmetry group, featuring a self-interaction with the quartic coupling constant $g$. We will be interested in the interacting critical regime of this model. In the free regime, $g=0$, the model sits at its Gaussian fixed point, where it is described by the MFT, with the bi-local kinetic action 
term analogous to (1.2),

$$
S_{0}=C(s) \int d^{d} x \int d^{d} y \frac{\phi_{i}(x) \phi_{i}(y)}{|x-y|^{d+s}} .
$$

This class of models is parametrized by the exponent $s$, characterizing the power-law long-range kinetic term in the action (2.1). The model (2.1) is defined for $s<2$, which ensures that the bi-local term is more relevant than the local kinetic term $\frac{1}{2} \partial \phi_{i} \partial \phi_{i}$. The latter dominates for $s>2$, when the theory crosses over to the local model (free short-range $O(N)$ vector model). In fact, when $s=2$, the model (2.1) is equivalent to the short-range $O(N)$ vector model, as it is easiest to see in momentum space. For $s=1$, the action (2.1) reduces, interestingly, to a boundary CFT (bCFT) with a free scalar field in the bulk, see [18] for some recent developments.

We will use the conventional choice of the factor $C(s)$ such that the propagator of $\phi_{i}$ in momentum space is normalized to unity (from now on we skip keeping track of the $O(N)$ indices and the associated Kronecker symbol whenever it does not cause an ambiguity),

$$
\langle\phi(p) \phi(q)\rangle=(2 \pi)^{d} \delta^{(d)}(p+q) \frac{1}{\left(p^{2}\right)^{\frac{s}{2}}} .
$$

Using the Fourier transform relation

$$
\int \frac{d^{d} k}{(2 \pi)^{d}} e^{i k \cdot x} \frac{1}{\left(k^{2}\right)^{\frac{d}{2}-\Delta}}=\frac{2^{2 \Delta-d}}{\pi^{\frac{d}{2}}} \frac{1}{A(\Delta)} \frac{1}{|x|^{2 \Delta}},
$$

where we defined

$$
A(\Delta)=\frac{\Gamma\left(\frac{d}{2}-\Delta\right)}{\Gamma(\Delta)},
$$

we obtain ${ }^{4}$

$$
C(s)=\frac{2^{s-1}}{\pi^{d / 2}} \frac{\Gamma\left(\frac{d+s}{2}\right)}{\Gamma\left(-\frac{s}{2}\right)} .
$$

The field $\phi$ has the dimension

$$
\Delta_{\phi}=\frac{d-s}{2},
$$

and its propagator in coordinate space has the form

$$
\langle\phi(x) \phi(0)\rangle=\frac{C_{\phi}}{|x|^{2 \Delta_{\phi}}},
$$

where we defined

$$
C_{\phi}=\frac{1}{2^{s} \pi^{\frac{d}{2}}} \frac{\Gamma\left(\frac{d-s}{2}\right)}{\Gamma\left(\frac{s}{2}\right)} .
$$

Perturbing the free theory (2.1) by a quartic self-interaction we obtain an interacting field theory with the action

$$
S=C(s) \int d^{d} x \int d^{d} y \frac{\phi(x) \phi(y)}{|x-y|^{d+s}}+\frac{g}{N} \int d^{d} x\left(\phi^{2}\right)^{2} .
$$

Setting $g=0$, we recover the free action (2.1). The quartic coupling constant $g$ is relevant when $s>d / 2$, and it triggers a flow to a fixed point in the IR. This can be established

\footnotetext{
${ }^{4}$ Notice that naively setting $s=2$ renders a singularity in $C(s)$. This artifact is lifted in momentum space.
} 
perturbatively by a Wilson-Fisher kind of $\epsilon$-expansion for $s=d / 2+\epsilon$. Together with the non-locality constraint $s<2$, this restricts us to consider $d<4$. When $s<d / 2$, the quartic coupling $g$ is irrelevant, and the theory flows to a fixed point in the UV limit, albeit the resulting model suffers from instabilities. This situation is analogous to the Wilson-Fisher fixed point of the short-range $O(N)$ vector model in $2<d<4$ and $4<d<6$ dimensions, respectively. When $s=1$, a UV-completion of the theory can be constructed [18], analogously to the higher-dimensional short-range $O(N)$ vector model [24].

It has been argued that the IR fixed point for $s>d / 2$ is reached in the entire range $1<d<4$, not merely in the perturbative regime around $d=2 s-2 \epsilon$. Importantly, one is interested in the physically relevant integer dimension $d=3$ (where the spectrum of the theory is expected to be unitary), and general allowed values for the exponent $s$. Some preliminary evidence in favor of this conclusion is devised in the large- $N$ limit using the Hubbard-Stratonovich transformation, analogously to the argument given in [25] for the short-range $O(N)$ vector models. Specifically, at the Gaussian f.p. the composite singlet field $\phi^{2}$ is a primary operator of dimension $\Delta_{\phi^{2}}=2 \Delta_{\phi}=d-s$. As we will review momentarily, employing the Hubbard-Stratonovich transformation one can demonstrate that the quartic interaction brings the theory to a new regime where $\phi^{2}$ exhibits a scaling behavior with the (leading order in $1 / N$ ) exponent $\Delta_{\phi^{2}}=\Delta_{\sigma}=s$, where $\sigma$ is the HubbardStratonovich field.

Using the Hubbard-Stratonovich transformation, the action (2.9) is equivalently rewritten as

$$
S=C(s) \int d^{d} x \int d^{d} y \frac{\phi(x) \phi(y)}{|x-y|^{d+s}}+\int d^{d} x\left(-\frac{1}{4 g} \sigma^{2}+\frac{1}{\sqrt{N}} \sigma \phi^{2}\right)
$$

The path integral over $\phi$ is now Gaussian, and integrating it out results in the effective action for $\sigma$

$$
S=\frac{N}{2} \int d^{d} x \int d^{d} y \operatorname{Tr} \log \left(\frac{C(s)}{|x-y|^{d+s}}+\frac{1}{\sqrt{N}} \sigma(x) \delta^{(d)}(x-y)\right)-\frac{1}{4 g} \int d^{d} x \sigma^{2} .
$$

Using the inverse propagator relation

$$
\int d^{d} y \frac{1}{|y|^{2 a}|x-y|^{2(d-a)}}=\pi^{d} A(a) A(d-a) \delta^{(d)}(x),
$$

and expanding the logarithm we obtain

$$
S=-C_{\phi}^{2} \int d^{d} x \int d^{d} y \frac{\sigma(x) \sigma(y)}{|x-y|^{2(d-s)}}-\frac{1}{4 g} \int d^{d} x \sigma^{2}+\mathcal{O}\left(\frac{1}{\sqrt{N}}\right),
$$

where we also used the definition (2.8).

For $s>d / 2$, and in the long-distance IR limit, the first term in the r.h.s. of $(2.13)$ dominates over the second term. ${ }^{5}$ The second term then drops out, and the $\langle\sigma \sigma\rangle$ propagator can be found from the first term using (2.12). As a result we obtain

$$
\langle\sigma(x) \sigma(0)\rangle=\frac{C_{\sigma}}{|x|^{2 \Delta_{s}}},
$$

\footnotetext{
${ }^{5}$ Analogously, when $s<d / 2$ the first term dominates over the second term in the UV limit.
} 
where the scaling dimension of the Hubbard-Stratonovich field $\sigma$ at the strongly-coupled regime in the IR is,

$$
\Delta_{\sigma}=s,
$$

and the propagator amplitude is found to be

$$
C_{\sigma}=-\frac{2^{2 s-1} \Gamma(d-s) \Gamma(s) \Gamma\left(\frac{s}{2}\right)^{2}}{\Gamma\left(\frac{d}{2}-s\right) \Gamma\left(s-\frac{d}{2}\right) \Gamma\left(\frac{d-s}{2}\right)^{2}} .
$$

As a consistency check, notice that for the scaling dimension (2.15) the second term in the r.h.s. of (2.13) is irrelevant.

In the effective action (2.13) we also omitted higher-order vertices for $\sigma$, which are suppressed in $1 / N$. We will encounter these vertices in our calculation of CFT data in this paper, where they will be explicitly represented diagrammatically via polygon graphs with internal $\phi$ propagator lines. These are constructed using the Feynman rules:
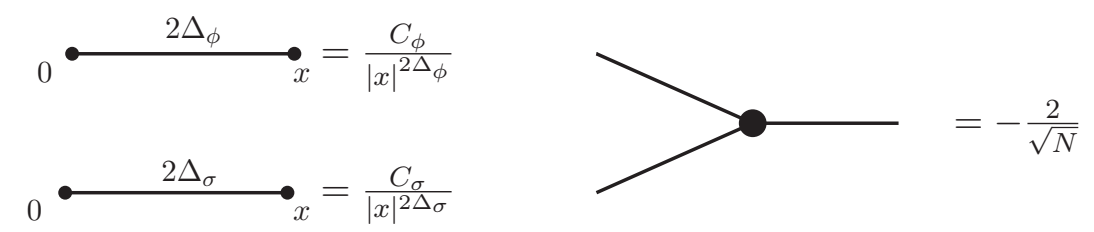

A propagator line with a generic exponent will be assumed to have a unit amplitude

$$
\underset{0}{\bullet} \stackrel{2 a}{\longrightarrow}=\frac{1}{|x|^{2 a}}
$$

In appendix A we collect some well-known identifies for conformal graphs in position space, that have been used for calculations in this paper.

\section{Two-point functions}

In this section we will calculate the $\langle\phi \phi\rangle$ and $\langle\sigma \sigma\rangle$ two-point functions of the fundamental field $\phi$ and the Hubbard-Stratonovich scalar field $\sigma$. Conformal invariance ensures that these two-point functions have the form

$$
\begin{aligned}
\langle\phi(x) \phi(0)\rangle & =\frac{C_{\phi}\left(1+A_{\phi}\right) \mu^{-2 \gamma_{\phi}}}{|x|^{2\left(\Delta_{\phi}+\gamma_{\phi}\right)}}, \\
\langle\sigma(x) \sigma(0)\rangle & =\frac{C_{\sigma}\left(1+A_{\sigma}\right) \mu^{-2 \gamma_{\sigma}}}{|x|^{2\left(\Delta_{\sigma}+\gamma_{\sigma}\right)}},
\end{aligned}
$$

where $\mu$ is an arbitrary mass scale, and $\Delta_{\phi, \sigma}$ are leading order dimensions at the longrange critical point, defined in section 2 . In this section we will calculate the two-point functions (3.1), (3.2) at the next-to-leading order in $1 / N$ expansion. In particular, we will reproduce the known expression for the anomalous dimensions $\gamma_{\sigma}$, and obtain new results for the relative corrections $A_{\phi, \sigma}$ to the amplitudes of these two-point functions. 
We will also demonstrate that the anomalous dimension $\gamma_{\phi}$ vanishes at the next-toleading order in $1 / N$. As discussed in section 2 , in fact we expect the engineering dimensions $\Delta_{\phi}$ of the field $\phi$ to be exact to all orders in $1 / N$, being fixed by the non-local kinetic term. However, our calculation of the two-point function $\langle\phi \phi\rangle$ is still useful, because we extract from it the relative amplitude correction $A_{\phi}$. While the propagator amplitude corrections are not observables, together with its counterpart $A_{\sigma}$, it will play an important part in our calculation in section 5 of the observable CFT data, such as the amplitudes of the $\langle\phi \phi \sigma\rangle$ and $\langle\sigma \sigma \sigma\rangle$ three-point functions and the related $\phi \phi$ and $\sigma \sigma$ OPE coefficients.

\section{$3.1\langle\phi \phi\rangle$}

It is easy to see that the only diagram contributing to the $\langle\phi \phi\rangle$ two-point function at the next-to-leading order in $1 / N$ is given by

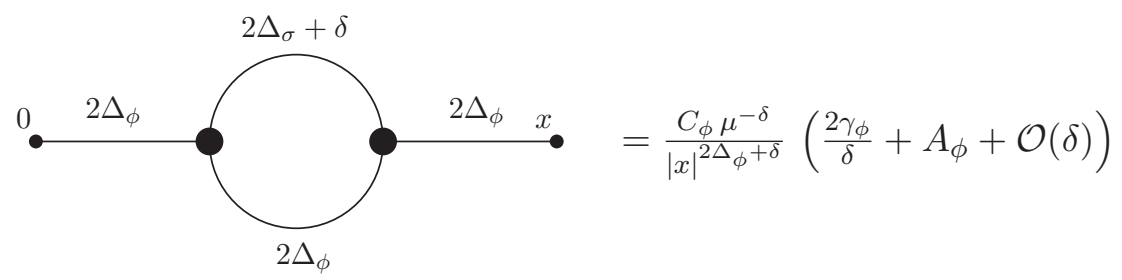

Here we have introduced an auxiliary regulator $\delta$ to the internal $\sigma$ line. Evaluating the diagram gives 6

$$
\langle\phi(x) \phi(0)\rangle \supset \frac{4}{N} \frac{C_{\phi}^{3} C_{\sigma}}{|x|^{2 \Delta_{\phi}}} U\left(\frac{d-s}{2}, \frac{d+s+\delta}{2},-\frac{\delta}{2}\right) U\left(\frac{d+\delta}{2}, \frac{d-s}{2}, \frac{s-\delta}{2}\right) \frac{1}{(\mu|x|)^{\delta}} .
$$

Expanding this expression around $\delta=0$ shows that it is in fact finite and $\gamma_{\phi}=0,{ }^{7}$ while

$$
A_{\phi}=\frac{1}{N} \frac{2^{d-s}(2 s-d) \Gamma(s) \sin \left(\frac{1}{2} \pi(d-2 s)\right) \Gamma\left(\frac{1}{2}(d-s+1)\right)}{\sqrt{\pi} s \Gamma\left(\frac{d+s}{2}\right) \sin \left(\frac{\pi s}{2}\right)}+\mathcal{O}\left(\frac{1}{N^{2}}\right) .
$$

\section{$3.2\langle\sigma \sigma\rangle$}

There are three diagrams that contribute to the $\langle\sigma \sigma\rangle$ correlation function at the nextto-leading order in $1 / N$, which we will label as $C_{\sigma \sigma}^{(a)}, a=1,2,3$. Just as in the case of $\langle\phi \phi\rangle$ two-point function, these diagrams are analogous to their counterparts in the shortrange critical $O(N)$ vector model, and therefore calculation sequences in these two models parallel each other. We refer the reader to [26] for a recent detailed description of such a calculation in the $O(N)$ vector model, while here we provide only a brief outline and a summary of results.

\footnotetext{
${ }^{6}$ In this paper we utilize the supset $\operatorname{sign} \supset$ to denote some of the terms which contribute to the expression on the 1.h.s. of this sign.

${ }^{7} \mathrm{~A}$ simple explanation of the vanishing anomalous dimension, $\gamma_{\phi}=0$, is that only local divergences can appear from the loops, which in turn are to be cancelled by local counter-terms. However, since the fields $\phi_{i}$ have a bi-local action, no local counter-terms can be obtained by the wave function renormalization of the $\phi_{i}$. Correspondingly, no divergences can appear in the loops of this renormalizable theory. In other words, the loops cannot induce wave-function renormalization of $\phi_{i}$.
} 
The total values of the anomalous dimension and the propagator amplitude correction at the next-to-leading order in $1 / N$ are obtained by summing the individual contributions from each of the three diagrams,

$$
\gamma_{\sigma}=\sum_{a=1}^{3} \gamma_{\sigma}^{(a)}, \quad A_{\sigma}=\sum_{a=1}^{3} A_{\sigma}^{(a)} .
$$

The first diagram contributing to $\langle\sigma \sigma\rangle$ is given by

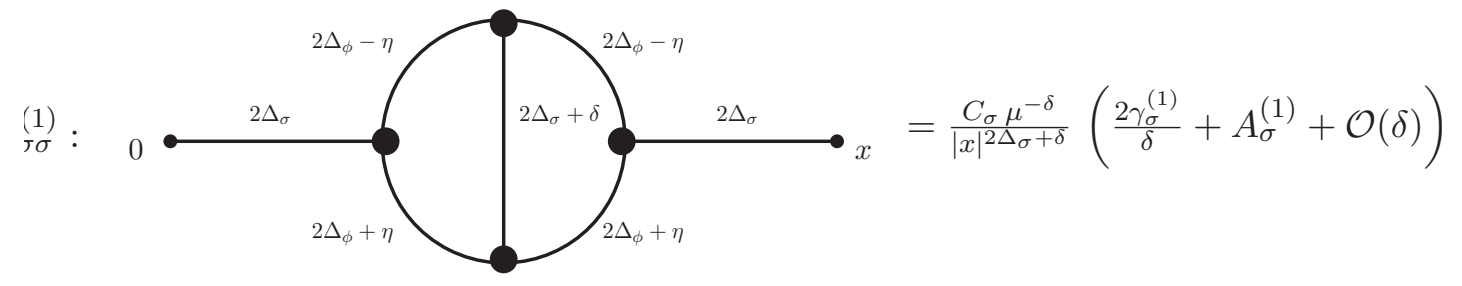

Here an auxiliary regulator $\eta=\mathcal{O}(\delta)$ does not affect the value of $C_{\sigma \sigma}^{(1)}$ in the $\delta \rightarrow 0$ limit $[17,27,28]$. Choosing $\eta=\delta / 2$ makes the diagram integrable via the uniqueness relation, rendering

$$
\begin{aligned}
\langle\sigma(x) \sigma(0)\rangle \supset & \frac{8}{N} \frac{C_{\phi}^{4} C_{\sigma}^{3}}{\left|x_{12}\right|^{2 \Delta_{\sigma}}} U\left(\Delta_{\phi}-\frac{\delta}{4}, \Delta_{\phi}-\frac{\delta}{4}, \Delta_{\sigma}+\frac{\delta}{2}\right) U\left(\frac{d+\delta}{2}, \frac{d+\delta}{2},-\delta\right) \\
& \times U\left(\Delta_{\sigma}, 2 \Delta_{\phi}+\frac{\delta}{2},-\frac{\delta}{2}\right) U\left(\Delta_{\sigma}, \frac{d+\delta}{2}, \frac{d-\delta}{2}-\Delta_{\sigma}\right) \frac{1}{(\mu|x|)^{\delta}}
\end{aligned}
$$

Expanding around $\delta=0$ one obtains the corresponding contributions of the diagram $C_{\sigma \sigma}^{(1)}$ to the $\gamma_{\sigma}$ and $A_{\sigma}{ }^{8}$

$$
\begin{aligned}
\gamma_{\sigma}^{(1)} & =-\frac{1}{N} \frac{4 \Gamma\left(\frac{s}{2}\right)^{2} \Gamma(d-s)}{\Gamma\left(\frac{d}{2}\right) \Gamma\left(\frac{d-s}{2}\right)^{2} \Gamma\left(s-\frac{d}{2}\right)} \\
A_{\sigma}^{(1)} & =\gamma_{\sigma}^{(1)}\left(\psi^{(0)}\left(\frac{d-s}{2}\right)-\psi^{(0)}(d-s) \psi^{(0)}\left(s-\frac{d}{2}\right)+\psi^{(0)}\left(\frac{s}{2}\right)\right) .
\end{aligned}
$$

Next, we consider contribution of the following diagram to $\langle\sigma \sigma\rangle$,

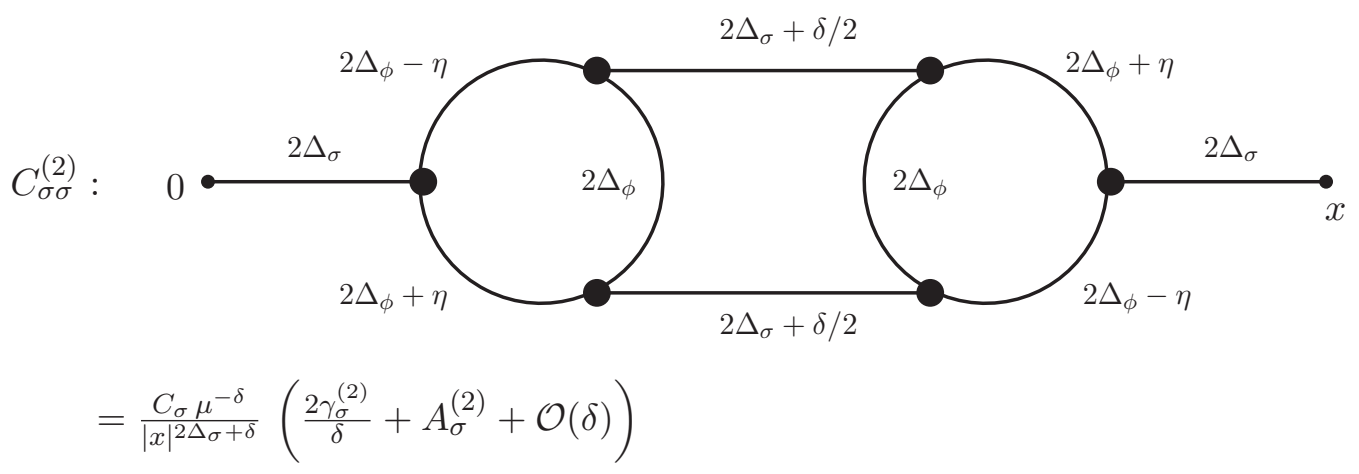

\footnotetext{
${ }^{8}$ In this paper we denote $n$th derivative of the digamma function as $\psi^{(n)}(x)$.
} 
Here we again introduced an auxiliary regulator $\eta=\mathcal{O}(\delta / 2)$ without affecting the value of this diagram in the $\delta \rightarrow 0$ limit. We choose $\eta=\delta / 2$, which renders some of the vertices unique and therefore integrable. Integrating over those vertices will result in a graph of the same topology to $C_{\sigma \sigma}^{(1)}$, which will require introducing yet again an auxiliary regulator $\eta^{\prime}=\mathcal{O}(\delta)$, in such a manner that the final answer in the $\delta \rightarrow 0$ limit will remain unaffected, while the uniqueness of the diagram becomes restored [17, 27, 28]. At the end, we obtain

$$
\begin{aligned}
\langle\sigma(x) \sigma(0)\rangle \supset & \frac{32}{N} \frac{C_{\phi}^{6} C_{\sigma}^{4}}{\left|x_{12}\right|^{2 \Delta_{\sigma}}} U\left(\Delta_{\phi}-\frac{\delta}{4}, \Delta_{\phi}, \Delta_{\sigma}+\frac{\delta}{4}\right)^{2} U\left(d-\frac{3 \Delta_{\sigma}}{2}-\frac{\delta}{4}, \frac{\Delta_{\sigma}}{2}-\frac{\delta}{4}, \Delta_{\sigma}+\frac{\delta}{2}\right) \quad(3.9) \\
& \times U\left(\frac{d+\delta}{2}, \frac{d+\delta}{2},-\delta\right) U\left(2 \Delta_{\phi}+\frac{\delta}{2}, \Delta_{\sigma},-\frac{\delta}{2}\right) U\left(\Delta_{\sigma}, \frac{d+\delta}{2}, \frac{d-\delta}{2}-\Delta_{\sigma}\right) \frac{1}{(\mu|x|)^{\delta}} .
\end{aligned}
$$

The contributions to anomalous dimension and the propagator amplitude are then given by

$$
\begin{aligned}
\gamma_{\sigma}^{(2)}= & \frac{1}{N} \frac{16 \Gamma\left(\frac{s}{2}\right)^{3} \Gamma\left(\frac{d}{2}-s\right) \Gamma(d-s)^{2} \Gamma\left(\frac{3 s}{2}-\frac{d}{2}\right)}{\Gamma\left(\frac{d}{2}\right) \Gamma(s) \Gamma\left(d-\frac{3 s}{2}\right) \Gamma\left(\frac{d-s}{2}\right)^{3} \Gamma\left(s-\frac{d}{2}\right)^{2}}, \\
A_{\sigma}^{(2)}= & \frac{\gamma_{\sigma}^{(2)}}{4}\left(3 \psi^{(0)}\left(\frac{d-s}{2}\right)+\psi^{(0)}\left(\frac{3 s}{2}-\frac{d}{2}\right)+\psi^{(0)}\left(d-\frac{3 s}{2}\right)-2 \psi^{(0)}\left(\frac{d}{2}-s\right)\right. \\
& \left.-2 \psi^{(0)}(d-s)-2\left(\psi^{(0)}\left(s-\frac{d}{2}\right)+\psi^{(0)}(s)\right)+3 \psi^{(0)}\left(\frac{s}{2}\right)\right) .
\end{aligned}
$$

Finally, the third diagram contributing to $\langle\sigma \sigma\rangle$ at the next-to-leading order in $1 / N$ is obtained by incorporating $1 / N$ correction to the $\langle\phi \phi\rangle$ sub-diagram of the leading-order $\phi$ bubble:

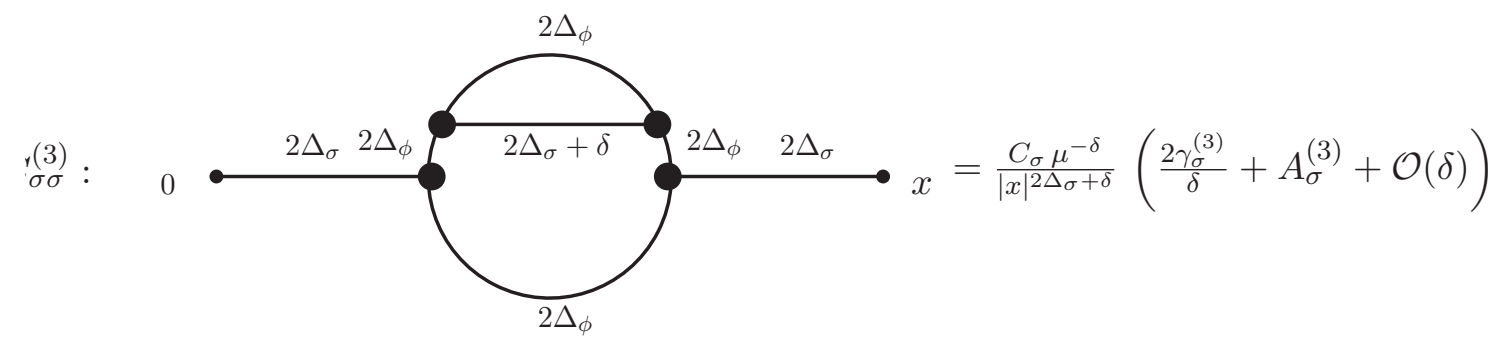

Such a correction to the $\phi$ propagator sub-diagram has been discussed in section 3.1, where it was established to be finite. Specifically, we obtain

$$
\begin{gathered}
\langle\sigma(x) \sigma(0)\rangle \supset \frac{16}{N} \frac{C_{\phi}^{4} C_{\sigma}^{3}}{|x|^{2 \Delta_{\sigma}}} U\left(\Delta_{\phi}, \Delta_{\phi}+\Delta_{\sigma}+\frac{\delta}{2},-\frac{\delta}{2}\right) U\left(\Delta_{\phi}, \frac{d+\delta}{2}, \frac{d-\delta}{2}-\Delta_{\phi}\right) \\
U\left(2 \Delta_{\phi}+\frac{\delta}{2}, \Delta_{\sigma},-\frac{\delta}{2}\right) U\left(\Delta_{\sigma}, \frac{d+\delta}{2}, \frac{d-\delta}{2}-\Delta_{\sigma}\right) \frac{1}{(\mu|x|)^{\delta}},
\end{gathered}
$$

expanding which around $\delta=0$ gives

$$
\begin{aligned}
& \gamma_{\sigma}^{(3)}=0 \\
& A_{\sigma}^{(3)}=\frac{1}{N} \frac{2^{d-s+1}(d-2 s) \csc \left(\frac{\pi s}{2}\right) \Gamma(s) \sin \left(\frac{1}{2} \pi(d-2 s)\right) \Gamma\left(\frac{1}{2}(d-s+1)\right)}{\sqrt{\pi} s \Gamma\left(\frac{d+s}{2}\right)} .
\end{aligned}
$$


Combining (3.5), (3.7), (3.8), (3.10), (3.11), (3.13), (3.14) we obtain the final answer for $\gamma_{\sigma}, A_{\sigma}$. Our result for $\gamma_{\sigma}$,

$$
\begin{aligned}
\gamma_{\sigma}= & -\frac{1}{N} \frac{4 \Gamma\left(\frac{s}{2}\right)^{2} \Gamma(d-s)}{\Gamma\left(\frac{d}{2}\right) \Gamma(s) \Gamma\left(d-\frac{3 s}{2}\right) \Gamma\left(\frac{d-s}{2}\right)^{3} \Gamma\left(s-\frac{d}{2}\right)^{2}}\left(\Gamma(s) \Gamma\left(d-\frac{3 s}{2}\right) \Gamma\left(\frac{d-s}{2}\right) \Gamma\left(s-\frac{d}{2}\right)\right. \\
& \left.-2 \Gamma\left(\frac{s}{2}\right) \Gamma\left(\frac{d}{2}-s\right) \Gamma(d-s) \Gamma\left(\frac{3 s}{2}-\frac{d}{2}\right)\right),
\end{aligned}
$$

agrees with $[17,18]$, while the expressions we obtained for the relative corrections $A_{\phi, \sigma}$ to the propagators of the $\phi$ and $\sigma$ are, to the best of our knowledge, new. While these are not observables, in combination with the $1 / N$ corrections to the effective cubic interaction vertices (conformal triangles) such as $\phi \phi \sigma$ and $\sigma \sigma \sigma$, they give amplitudes of the three-point functions (e.g., $\langle\phi \phi \sigma\rangle$ and $\langle\sigma \sigma \sigma\rangle$ ), which determine the OPE coefficients, and are a part of CFT data.

\section{Conformal invariance and composite operators}

In this section, we will discuss conformal invariance of the long-range fixed point at the level of two-point correlation functions of composite operators such as $\sigma^{n}, n>1$, and $\sigma \phi$. Recall that in the short-range critical $O(N)$ vector model composite operators of the kind $\hat{\sigma}^{n}, n>1,{ }^{9}$ are usually scaling operators $[29,30] .{ }^{10}$ These operators, therefore, have a fixed scaling dimension, and further acquire an anomalous dimension at the fixed point. However, such operators are usually not conformal primaries in the theory. In fact, since $\hat{\sigma}$ has a fixed scaling dimension, $\Delta_{\hat{\sigma}}=2$ for any $2<d<4$, it mixes with other descendent operators of the same dimension, created by replacing some of the $\hat{\sigma}$ fields with derivatives. The resulting operators furnish true conformal primaries in the theory [29, 30].

The spectrum of long-range CFTs is slightly different. The Hubbard-Stratonovich field $\sigma$ in such a theory has a leading scaling dimension $\Delta_{\sigma}=s$, which is generally a non-integer number. It implies immediately that one cannot create local descendent operators with leading-order scaling dimensions equal to that of $\sigma^{n}$, by simply replacing some of the $\sigma$ fields with derivatives. Such composite operators, therefore, do not mix with any other operators, and are expected to be conformal primaries by themselves. ${ }^{11}$ Below, we will test these statements at the level of two-point correlators of some composite operators. If the composite operators are indeed conformal primaries in the long-range CFT, we will find that the two point function of any composite operator with itself exhibits a power-law scaling behavior, while its correlators with other operators of different scaling dimensions are exactly zero.

\section{$4.1\left\langle\sigma^{2} \sigma^{2}\right\rangle$}

We begin by calculating the two-point correlation function of the composite operator $\sigma^{2}$. Starting from the assumption that $\sigma^{2}$ is a scaling operator, as we will explicitly establish

\footnotetext{
${ }^{9}$ We use $\hat{\sigma}$ to denote the Hubbard-Stratonovich field in the short-range critical $O(N)$ vector model, while its counterpart in the long-range model is denoted as $\sigma$.

${ }^{10}$ See [31] for an extensive earlier work on the formalism of scaling operators.

${ }^{11}$ The case of $s=1$ needs a separate discussion.
} 
below at the next-to-leading order in $1 / N$ expansion, its two-point function is expected to have the form

$$
\left\langle\sigma^{2}(x) \sigma^{2}(0)\right\rangle=\frac{C_{\sigma^{2}}\left(1+A_{\sigma^{2}}\right)}{|x|^{2\left(\Delta_{\sigma^{2}}+\gamma_{\sigma^{2}}\right)}},
$$

where we separated the amplitude into the leading part $C_{\sigma^{2}}$ and its relative $1 / N$ corrections $A_{\sigma^{2}}$. At the same time, $\Delta_{\sigma^{2}}$ stands for the leading order scaling dimension, while $\gamma_{\sigma^{2}}$ is the anomalous dimension contribution. In the large- $N$ limit the $\left\langle\sigma^{2} \sigma^{2}\right\rangle$ propagator is determined by the diagram

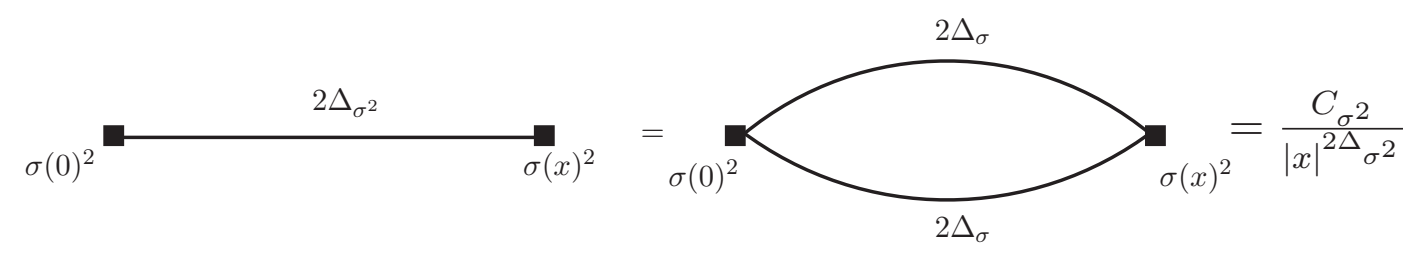

from which one easily finds

$$
C_{\sigma^{2}}=2 C_{\sigma}^{2}, \quad \Delta_{\sigma^{2}}=2 \Delta_{\sigma}=2 s .
$$

There are four diagrams that contribute to the $\left\langle\sigma^{2} \sigma^{2}\right\rangle$ propagator at the next-to-leading order in $1 / N$. The first contribution is simply due to the $1 / N$ corrections to the propagators of $\sigma$ in the leading order diagram. We calculated these corrections in section 3.2, and will denote their total contribution with a gray blob:

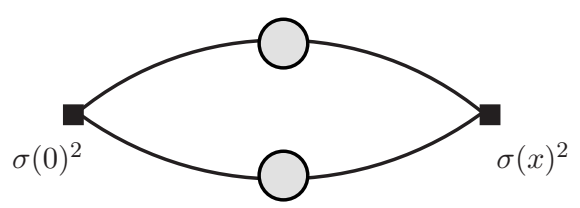

This diagram includes the leading-order $\left\langle\sigma^{2} \sigma^{2}\right\rangle$ propagator, as well as some contributions to $\gamma_{\sigma^{2}}$ and $A_{\sigma^{2}}$,

$$
\left\langle\sigma^{2}(x) \sigma^{2}(0)\right\rangle \supset \frac{C_{\sigma^{2}}\left(1+A_{\sigma}\right)^{2}}{|x|^{4 \Delta_{\sigma}}}\left(1-4 \gamma_{\sigma} \log (|x| \mu)\right) .
$$

Here, and in what follows, we are going to ignore the finite terms such as $2 A_{\sigma}$ in (4.3), and focus only on the singular terms, that contribute to the anomalous dimension $\gamma_{\sigma^{2}}$. This will greatly simplify the calculation, since we can avoid introducing the regulator $\delta$, and therefore easily take all of the unique integrals. The last logarithmically divergent integral is simply replaced with $\left(2 \pi^{\frac{d}{2}} / \Gamma(d / 2)\right) \log (\mu)$, where $\mu$ is IR mass scale [32, 33].

The second contribution to $\left\langle\sigma^{2} \sigma^{2}\right\rangle$ at the next-to-leading order in $1 / N$ is due to insertions of two cubic $\sigma$ vertices into the leading order diagram: ${ }^{12}$

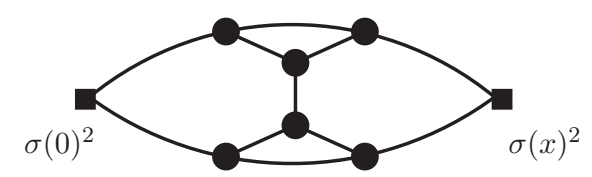

\footnotetext{
${ }^{12}$ To lighten up the notation, we skip labeling exponents on diagrams in the rest of this subsection.
} 
Evaluating this diagram gives

$$
\begin{aligned}
\left\langle\sigma^{2}(x) \sigma^{2}(0)\right\rangle \supset & \frac{128}{N} \frac{C_{\phi}^{6} C_{\sigma}^{5}}{|x|^{2 \Delta_{\sigma^{2}}}} U\left(s, \frac{d-s}{2}, \frac{d-s}{2}\right)^{2} U\left(\frac{s}{2}, s, d-\frac{3 s}{2}\right) \\
& \times U\left(\frac{d-s}{2}, d-\frac{3 s}{2}, 2 s-\frac{d}{2}\right) U(s, s, d-2 s) \frac{4 \pi^{d / 2}}{\Gamma(d / 2)} \log (|x| \mu) .
\end{aligned}
$$

The third and fourth diagrams are obtained due to insertion of a single quartic vertex into the propagator. The corresponding planar diagram is given by

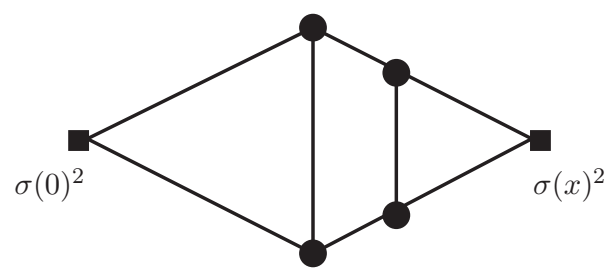

and contributes

$$
\left\langle\sigma^{2}(x) \sigma^{2}(0)\right\rangle \supset \frac{32}{N} \frac{C_{\phi}^{4} C_{\sigma}^{4}}{|x|^{2 \Delta_{\sigma^{2}}}} U\left(s, \frac{d-s}{2}, \frac{d-s}{2}\right)^{2} U\left(\frac{s}{2}, \frac{3 s}{2}, d-2 s\right) \frac{4 \pi^{d / 2}}{\Gamma(d / 2)} \log (|x| \mu),
$$

while the non-planar diagram

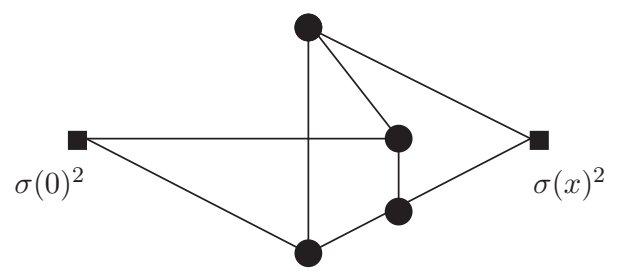

contributes

$$
\left\langle\sigma^{2}(x) \sigma^{2}(0)\right\rangle \supset \frac{16}{N} \frac{C_{\phi}^{4} C_{\sigma}^{4}}{|x|^{2 \Delta_{\sigma^{2}}}} U\left(s, \frac{d-s}{2}, \frac{d-s}{2}\right)^{2} U(s, s, d-2 s) \frac{4 \pi^{d / 2}}{\Gamma(d / 2)} \log (|x| \mu) .
$$

Combining (4.3), (4.4), (4.5) and (4.6) we obtain the anomalous dimension of $\sigma^{2}$ at the next-to-leading order in $1 / N$,

$$
\begin{aligned}
\gamma_{\sigma^{2}}= & -\frac{1}{N} \frac{4 \Gamma\left(\frac{s}{2}\right)^{2} \Gamma(d-s)}{\Gamma\left(\frac{d}{2}\right) \Gamma(s)^{2} \Gamma\left(\frac{3 s}{2}\right) \Gamma(d-2 s) \Gamma\left(d-\frac{3 s}{2}\right)^{2} \Gamma\left(\frac{d-s}{2}\right)^{4} \Gamma\left(s-\frac{d}{2}\right)^{3}} \\
& \times\left(\Gamma\left(\frac{s}{2}\right) \Gamma(d-s) \Gamma\left(s-\frac{d}{2}\right) \Gamma\left(2 s-\frac{d}{2}\right) \Gamma\left(d-\frac{3 s}{2}\right)^{2}\right. \\
& \times\left(\Gamma\left(\frac{s}{2}\right) \Gamma\left(\frac{3 s}{2}\right) \Gamma\left(\frac{d}{2}-s\right)^{2}+2 \Gamma(s)^{2} \Gamma\left(\frac{1}{2}(d-3 s)\right) \Gamma\left(\frac{d-s}{2}\right)\right) \\
& +2 \Gamma\left(\frac{3 s}{2}\right) \Gamma(d-2 s)\left(\Gamma(s)^{2} \Gamma\left(d-\frac{3 s}{2}\right)^{2} \Gamma\left(\frac{d-s}{2}\right)^{2} \Gamma\left(s-\frac{d}{2}\right)^{2}\right. \\
& -2 \Gamma\left(\frac{s}{2}\right) \Gamma(s) \Gamma\left(d-\frac{3 s}{2}\right) \Gamma\left(\frac{d}{2}-s\right) \Gamma\left(\frac{d-s}{2}\right) \Gamma(d-s) \Gamma\left(\frac{3 s}{2}-\frac{d}{2}\right) \Gamma\left(s-\frac{d}{2}\right) \\
& \left.\left.-2 \Gamma\left(\frac{s}{2}\right)^{2} \Gamma\left(\frac{d}{2}-s\right)^{2} \Gamma(d-s)^{2} \Gamma\left(\frac{3 s}{2}-\frac{d}{2}\right)^{2}\right)\right)
\end{aligned}
$$


A quick consistency check of this expression is given by $\left.\gamma_{\sigma^{2}}\right|_{s=d / 2}=0$, since at $s=d / 2$ the long-range CFT becomes free. Another check is given by continuity at the long-rangeshort-range crossover point $s=s_{\star}$, which demands $\left.\gamma_{\sigma^{2}}\right|_{s=s_{\star}}=\gamma_{\hat{\sigma}^{2}}$, where $\gamma_{\hat{\sigma}^{2}}$ is anomalous dimension of the composite operator $\hat{\sigma}^{2}$ in short-range $O(N)$ vector model CFT. This crossover will be discussed in more detail in section 6 .

\section{$4.2\left\langle\sigma^{n} \sigma^{n}\right\rangle$}

The $\left\langle\sigma^{n} \sigma^{n}\right\rangle$ propagator of the composite field $\sigma^{n}$ (where $n \in \mathbb{N}$ ) at the next-to-leading order in $1 / N$ is determined by the diagrams
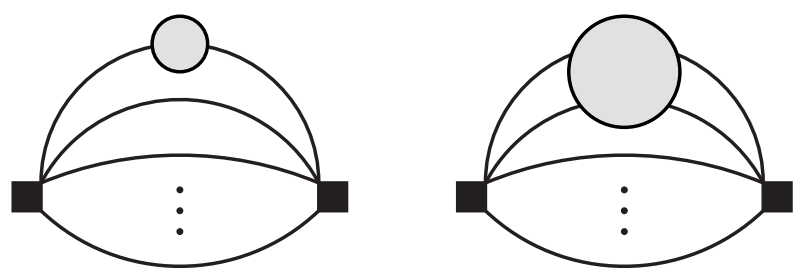

Here we used gray blobs to denote dressed $\langle\sigma \sigma\rangle$ and $\left\langle\sigma^{2} \sigma^{2}\right\rangle$ propagators. The anomalous dimension $\gamma_{\sigma^{n}}$ is therefore determined only by $\gamma_{\sigma}$ and $\gamma_{\sigma^{2}}$. Further corrections contribute only at higher orders in $1 / N$. The value of $\gamma_{\sigma^{n}}$ is then found to be,

$$
\gamma_{\sigma^{n}}=n(2-n) \gamma_{\sigma}+\frac{n(n-1)}{2} \gamma_{\sigma^{2}}
$$

The combinatorics involved is simple: we first account for the anomalous dimension corresponding to $n$ propagators of $\sigma$, the second term then computes the propagator corrections for $\sigma^{2}$ (there are $n(n-1) / 2$ such corrections). To avoid over-counting, we subtract $2 \gamma_{\sigma}$ from each $\sigma^{2}$ correction, which ultimately gives the first term.

\subsection{The $\left\langle\sigma^{2} \sigma\right\rangle$ correlator and conformal invariance}

At the short-range IR fixed point of the $O(N)$ vector model in $2<d<4$ dimensions the composite operator $\hat{\sigma}^{2}$ is in general not a conformal primary [29, 30]. For instance, in general $d$ the correlator $\left\langle\hat{\sigma}^{2} \hat{\sigma}\right\rangle$ is non-vanishing, while the scaling dimensions of $\hat{\sigma}$ and $\hat{\sigma}^{2}$ are manifestly different. However, the leading order scaling dimensions of the operators $\hat{\sigma}^{2}$ and $\partial^{2} \hat{\sigma}$ are the same, and therefore due to the non-vanishing $\left\langle\hat{\sigma}^{2} \hat{\sigma}\right\rangle$ these operators can mix. In fact they do, and result in a primary operator of the form $\hat{\sigma}^{2}+\alpha \partial^{2} \hat{\sigma}$ for a certain $\alpha=\mathcal{O}(1 / \sqrt{N})[29,30] .{ }^{13}$

However, in the long-range CFT, due to the non-integer scaling dimension of $\sigma^{2}$, we do not expect it to mix with any other descendent operator. In fact, we expect it to be a conformal primary in the theory with $1 \leq d<4$, and for any allowed value of $s$ in the long-range CFT region. One immediate manifestation of this fact should be borne out in the correlator $\left\langle\sigma^{2} \sigma\right\rangle$, which is expected to vanish. In this section, we perform this check explicitly, working at the leading order in $1 / N$.

\footnotetext{
${ }^{13}$ Note, however, that the mixing coefficient $\alpha$ vanishes for the integer-valued $d=2,3,4$.
} 
To the leading order, the $\left\langle\sigma^{2} \sigma\right\rangle$ correlator is determined by the kite diagram with a $\phi$ loop in the middle:

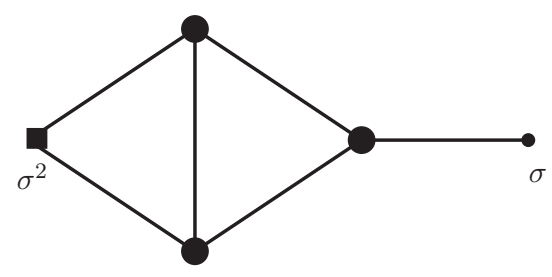

One way to compute it is to replace the $\sigma^{2} \sigma \sigma$, and $\sigma \sigma \sigma$ vertices with their respective conformal triangles: ${ }^{14}$

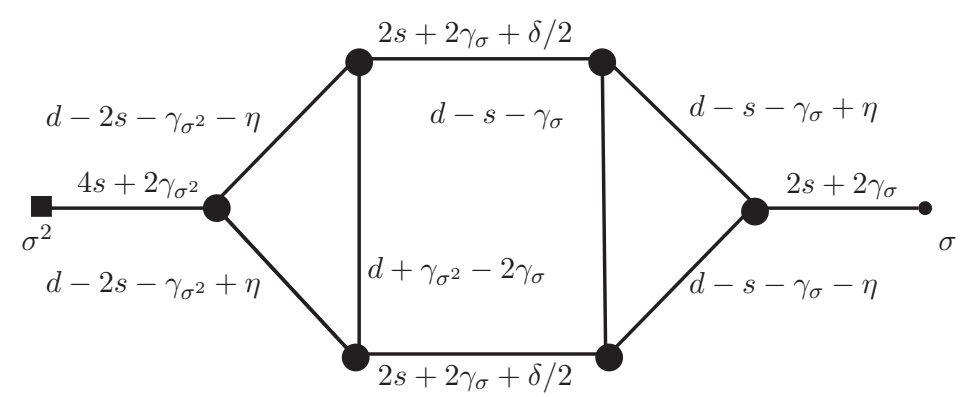

Integrating over the internal vertices, while introducing auxiliary regulators wherever necessary (such as choosing $\eta=\delta / 2$ in the diagram above), we arrive at the final result given by

$$
\begin{aligned}
& \left\langle\sigma^{2}(x) \sigma(0)\right\rangle=\frac{1}{2}\left(-6 Z_{\sigma \sigma \sigma}^{(0)}\right)\left(-2 Z_{\sigma^{2} \sigma \sigma}^{(0)}\right) C_{\sigma^{2}} C_{\sigma}^{3} U\left(\frac{d-\gamma_{\sigma^{2}}}{2}-s-\frac{\delta}{4}, s+\gamma_{\sigma}+\frac{\delta}{4}, \frac{d+\gamma_{\sigma^{2}}}{2}-\gamma_{\sigma}\right) \\
& \quad \times U\left(\frac{d-s}{2}, \frac{d-s}{2}-\frac{\delta}{4}, s+\frac{\delta}{4}\right) U\left(d-\frac{3 s}{2}, \frac{3 s}{2}+\frac{\delta}{2},-\frac{\delta}{2}\right) U\left(s, \frac{d+s+\delta}{2}, \frac{d-3 s-\delta}{2}\right) \\
& \quad \times U\left(d-2 s, \frac{3 s+\delta}{2}, \frac{s-\delta}{2}\right) U\left(2 s, \frac{d-s+\delta}{2}, \frac{d-3 s-\delta}{2}\right) \frac{\mu^{-\delta}}{|x|^{3 s+\delta}} .
\end{aligned}
$$

Here the large- $N$ amplitudes of the $\sigma^{2} \sigma \sigma$ and $\sigma \sigma \sigma$ conformal triangles are given by ${ }^{15}$

$$
Z_{\sigma^{2} \sigma \sigma}^{(0)}=-\frac{1}{2} \frac{C_{\sigma^{2} \sigma \sigma}^{(0)}}{C_{\sigma^{2}} C_{\sigma}^{2} u_{\sigma^{2} \sigma \sigma}^{(0)}}=-\frac{1}{C_{\sigma^{2}} u_{\sigma^{2} \sigma \sigma}^{(0)}}, \quad Z_{\sigma \sigma \sigma}^{(0)}=\frac{4 C_{\phi}^{3}}{3},
$$

where we have used the leading-order amplitude of the $\left\langle\sigma^{2} \sigma \sigma\right\rangle$ three-point function

$$
C_{\sigma^{2} \sigma \sigma}^{(0)}=2 C_{\sigma}^{2},
$$

as well as the propagator amplitude (4.2). The expression for $u_{\sigma^{2} \sigma \sigma}^{(0)}$ is obtained by integrating over the vertices of the conformal triangle for $\sigma^{2} \sigma \sigma$, and then keeping only the

\footnotetext{
${ }^{14}$ It should be noted that we introduce the conformal triangles to regulate the diagram even though it is finite. However, the diagram is superficially divergent, and in order to calculate it, we choose to re-write it in an equivalent form that possesses internal $\sigma$ lines. The diagram is then regularized by a small shift $\delta$ of the exponents of internal $\sigma$ propagators, even though the $\delta \rightarrow 0$ limit of such a diagram is finite.

${ }^{15}$ See section 5 for detailed discussion of conformal triangles in the long-range critical vector model.
} 
leading large $N$ terms ${ }^{16}$

$$
u_{\sigma^{2} \sigma \sigma}^{(0)}=\frac{2}{2 \gamma_{\sigma}-\gamma_{\sigma^{2}}} \frac{\pi^{3 d / 2} \Gamma\left(\frac{d}{2}-2 s\right) \Gamma\left(2 s-\frac{d}{2}\right)}{\Gamma\left(\frac{d}{2}\right) \Gamma(2 s) \Gamma(d-2 s)} .
$$

The factor of $2 \gamma_{\sigma}-\gamma_{\sigma^{2}}$ is cancelled precisely by a leading large $N$ expansion of the only factor in (4.9) whose dependence on the anomalous dimensions we retain for the sake of regularizing the corresponding $U$ function, namely

$$
U\left(\frac{d-\gamma_{\sigma^{2}}}{2}-s-\frac{\delta}{4}, s+\gamma_{\sigma}+\frac{\delta}{4}, \frac{d+\gamma_{\sigma^{2}}}{2}-\gamma_{\sigma}\right)=\frac{2 \pi^{d / 2}}{\left(2 \gamma_{\sigma}-\gamma_{\sigma^{2}}\right) \Gamma\left(\frac{d}{2}\right)}+\mathcal{O}\left(N^{0}\right) .
$$

Combining everything together and taking the limit $\delta \rightarrow 0$, we obtain

$$
\left\langle\sigma^{2}(x) \sigma(0)\right\rangle=0+\mathcal{O}\left(\frac{1}{N^{3 / 2}}\right) .
$$

The vanishing of the leading order correlator between $\sigma$ and $\sigma^{2}$ provides further evidence that $\sigma^{2}$ is indeed a primary at the long-range fixed point, and that the fixed point in fact enjoys the full conformal symmetry. As an additional check, one can compute the first sub-leading order correction in the $1 / N$ expansion, and show that it also vanishes for any $1 \leq d<4$, and the corresponding allowed values of $s$ for the long-range CFT region.

\section{$4.4\langle\sigma \phi \sigma \phi\rangle$}

In this section we will discuss the operator $\sigma \phi$, which will play an important role in section 7, where we will develop the $O(N)$ generalization of the short-range-long-range duality proposal of [11]. This composite operator has two constituents, one of which, $\sigma$, possesses an anomalous dimension. However, interestingly enough, the composite operator $\sigma \phi$ itself does not acquire anomalous dimension to all orders in $1 / N$. This exact result follows immediately from the $\phi$ e.o.m. due to the action (2.10), relating dimension of $\sigma \phi$ to the dimension of $\phi$ :

$$
\sigma \phi=-\sqrt{N} C(s) \int d^{d} y \frac{\phi(y)}{|x-y|^{d+s}} .
$$

Using the $\langle\phi \phi\rangle$ two-point function (3.1), while taking into account $\gamma_{\phi}=0$, we obtain

$$
\langle\sigma \phi(x) \sigma \phi(0)\rangle=N C(s)^{2} C_{\phi}\left(1+A_{\phi}\right) \pi^{d} A\left(\frac{d-s}{2}\right) A\left(\frac{d+s}{2}\right) \frac{1}{|x|^{d+s}},
$$

where we also took advantage of the identity (2.12). We will be ignoring the $1 / N$ corrections to the propagator amplitude, while noticing that the scaling dimension of the composite operator $\sigma \phi$ is given by

$$
\Delta_{\sigma \phi}=\frac{d+s}{2},
$$

\footnotetext{
${ }^{16}$ See [34] for analogous calculation in the short-range $O(N)$ vector model, where the corresponding $s^{2} s s$ conformal triangle is calculated at the next-to-leading order in $1 / N$ expansion.
} 
exactly to all orders in $1 / N$, despite the fact that the constituent $\sigma$ has a non-trivial anomalous dimension.

As a consistency check, the relation (4.17) can be quickly verified at the next-to-leading order in $1 / N$ expansion. The first contribution is obtained by dressing of the internal $\sigma$ and $\phi$ lines of the leading-order diagram:

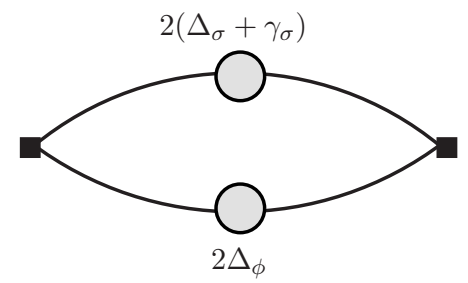

Its contribution to the anomalous dimension is then given by

$$
\gamma_{\sigma \phi}^{(1)}=\gamma_{\sigma} .
$$

Next, we have the contribution due to the effective $\sigma \sigma \sigma$ vertex inserted into the leadingorder diagram:

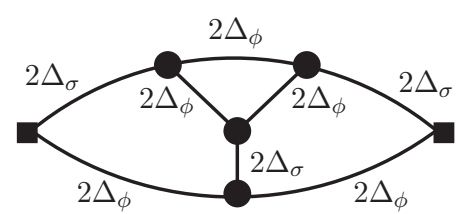

This diagram is divergent. Since we are only interested in anomalous dimensions, we can regularize it by simply extracting the logarithmically divergent term of the last integral. ${ }^{17}$ As a result we obtain contribution to the anomalous dimension given by

$$
\gamma_{\sigma \phi}^{(2)}=-\frac{16}{N} C_{\phi}^{4} C_{\sigma}^{2} \frac{2 \pi^{\frac{d}{2}}}{\Gamma\left(\frac{d}{2}\right)} U\left(\frac{d-s}{2}, \frac{d-s}{2}, s\right)^{2} U\left(s, \frac{s}{2}, d-\frac{3 s}{2}\right) .
$$

Finally, we have contribution from the following diagram:

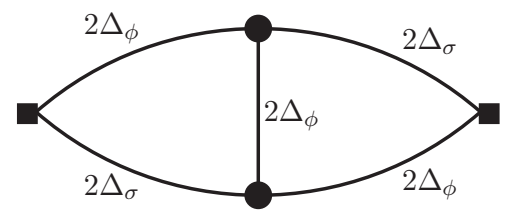

Regularizing it using (4.19) we obtain

$$
\gamma_{\sigma \phi}^{(3)}=-\frac{8}{N} C_{\phi}^{2} C_{\sigma} \frac{2 \pi^{\frac{d}{2}}}{\Gamma\left(\frac{d}{2}\right)} U\left(\frac{d-s}{2}, \frac{d-s}{2}, s\right) .
$$

${ }^{17}$ This can be done by using the relation

$$
\int d^{d} y \frac{1}{|y|^{d}|x-y|^{d}} \supset 2 \frac{2 \pi^{\frac{d}{2}}}{\Gamma\left(\frac{d}{2}\right)} \log (\mu) \frac{1}{|x|^{d}} .
$$


Combining (4.18), (4.20), (4.21), while taking into account $\gamma_{\sigma}$ given by (3.15), ${ }^{18}$ we arrive at

$$
\gamma_{\sigma \phi}=\gamma_{\sigma \phi}^{(1)}+\gamma_{\sigma \phi}^{(2)}+\gamma_{\sigma \phi}^{(3)}=0+\mathcal{O}\left(\frac{1}{N^{2}}\right)
$$

in agreement with the general argument given above.

\section{OPE coefficients}

We now proceed to calculating various OPE coefficients in the long-range $O(N)$ critical vector model. In section 5.1 we calculate the $\langle\phi \phi \sigma\rangle$ three-point function, and determine its amplitude at the next-to-leading order in $1 / N$ expansion. Such a calculation requires knowing the next-to-leading order corrections to the $\phi \phi \sigma$ interaction vertex. We calculate this non-local effective vertex using the background field method [32]. In section 5.2 we calculate the $\langle\sigma \sigma \sigma\rangle$ three-point function at the leading order in $1 / N$ expansion.

\section{$5.1\langle\phi \phi \sigma\rangle$}

In this section we will calculate the $\langle\phi \phi \sigma\rangle$ correlation function at the next-to-leading order in $1 / N$ expansion. Due to conformal symmetry, this three-point function has the form

$$
\left\langle\phi\left(x_{1}\right) \phi\left(x_{2}\right) \sigma\left(x_{3}\right)\right\rangle=\frac{C_{\phi \phi \sigma}^{(0)}\left(1+\delta C_{\phi \phi \sigma}\right) \mu^{-\gamma_{\sigma}}}{\left(\left|x_{13}\right|\left|x_{23}\right|\right)^{\Delta_{\sigma}+\gamma_{\sigma}}\left|x_{12}\right|^{2 \Delta_{\phi}-\Delta_{\sigma}-\gamma_{\sigma}}},
$$

where $\mu$ is an arbitrary mass scale. The amplitude of the three-point function (5.1) was separated into the leading order factor of $C_{\phi \phi \sigma}^{(0)}$ and the relative $1 / N$ corrections to it, $\delta C_{\phi \phi \sigma}=\mathcal{O}(1 / N)$. We also took into account that the anomalous dimension of $\phi$ vanishes, and therefore its total dimension is given by $\Delta_{\phi}$.

Carrying out the calculation of (5.1) in this section, we will reproduce the anomalous dimension $\gamma_{\sigma}$, and derive the leading order amplitude $C_{\phi \phi \sigma}^{(0)}$ and the next-to-leading order contribution to $\delta C_{\phi \phi \sigma}$. In the process, we will introduce and calculate the $\phi \phi \sigma$ conformal triangle, at the next-to-leading order in $1 / N$, representing the corresponding non-local vertex in the effective action.

It is customary to use conventions in which position-space propagators of the fields in the considered correlation function are normalized to unity. This can be achieved by the corresponding rescaling of the fields,

$$
\phi \rightarrow \sqrt{C_{\phi}\left(1+A_{\phi}\right)} \phi, \quad \sigma \rightarrow \sqrt{C_{\sigma}\left(1+A_{\sigma}\right)} \sigma .
$$

In particular, such a normalization provides a renormalization scheme choice related to the freedom of redefining the scale $\mu$ by a constant factor. We will denote the amplitudes of the correlation functions with so-normalized fields with a bar, e.g., $\bar{C}_{\phi \phi \sigma}^{(0)}$ and $\delta \bar{C}_{\phi \phi \sigma}$ for the three-point function (5.1) in which all of the fields have been normalized according to (5.2).

\footnotetext{
${ }^{18}$ Alternatively, this calculation can be viewed as a consistency check for the value of $\gamma_{\sigma}$.
} 
The leading order amplitude $C_{\phi \phi s}^{(0)}$ can be easily found from the tree-level diagram

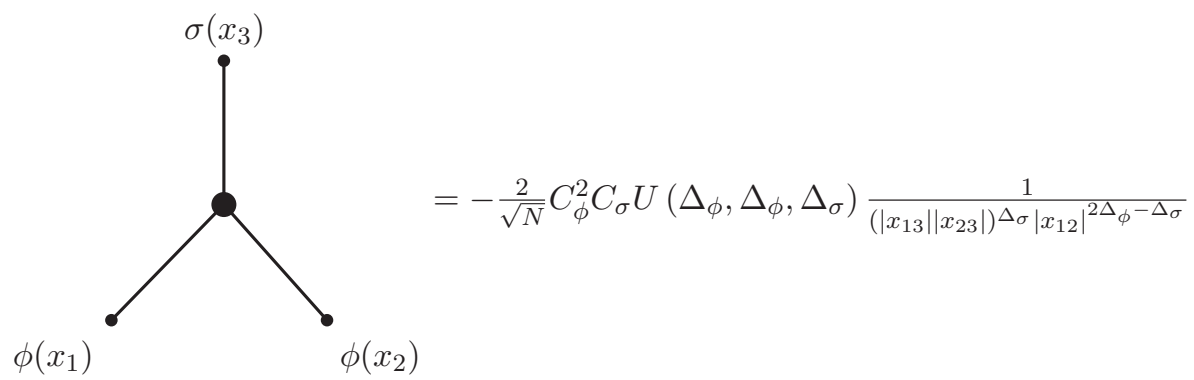

For the fields normalized according to (5.2) we obtain the leading-order amplitude

$$
\begin{aligned}
\bar{C}_{\phi \phi \sigma}^{(0)} & =-\frac{2}{\sqrt{N}} C_{\phi} C_{\sigma}^{\frac{1}{2}} U\left(\Delta_{\phi}, \Delta_{\phi}, \Delta_{\sigma}\right) \\
& =-\frac{1}{\sqrt{N}} \frac{\Gamma\left(\frac{s}{2}\right)^{2} \Gamma\left(\frac{d}{2}-s\right) \sqrt{(d-2 s) \Gamma(s) \sin \left(\frac{1}{2} \pi(d-2 s)\right) \Gamma(d-s)}}{\sqrt{\pi} \Gamma(s) \Gamma\left(\frac{d-s}{2}\right)^{2}} .
\end{aligned}
$$

To determine correction $\delta C_{\phi \phi \sigma}$ to the amplitude of the $\langle\phi \phi \sigma\rangle$ three-point function, we begin by calculating the corresponding $\phi \phi \sigma$ conformal triangle (see [35] for the original discussion of conformal triangles in CFTs). In terms of the effective action, such a conformal triangle gives a diagrammatic representation of the non-local interaction term

$$
S_{\text {eff }} \supset \frac{Z_{\phi \phi \sigma}}{\sqrt{N}} \mu^{\gamma_{\sigma}} \int d^{d} x_{1,2,3} \frac{\phi\left(x_{1}\right) \phi\left(x_{2}\right) \sigma\left(x_{3}\right)}{\left(\left|x_{13}\right|\left|x_{23}\right|\right)^{2 \alpha}\left|x_{12}\right|^{2 \beta}},
$$

where we denoted

$$
\alpha=\frac{d-s-\gamma_{\sigma}}{2}, \quad \beta=s+\frac{\gamma_{\sigma}}{2} .
$$

The amplitude of the conformal triangle admits the $1 / N$ expansion, $Z_{\phi \phi \sigma}=Z_{\phi \phi \sigma}^{(0)}(1+$ $\left.\delta Z_{\phi \phi \sigma}\right)$. Here the leading order amplitude $Z_{\phi \phi \sigma}^{(0)}=\mathcal{O}(1)$ is constrained by the requirement that the classical interaction term $\frac{1}{\sqrt{N}} \phi^{2} \sigma$ is reproduced in the large- $N$ limit. Incorporating the next-to-leading order correction, and taking into account the vertex counter-term contribution, we obtain the following diagrammatic equation for the $\phi \phi \sigma$ conformal triangle:
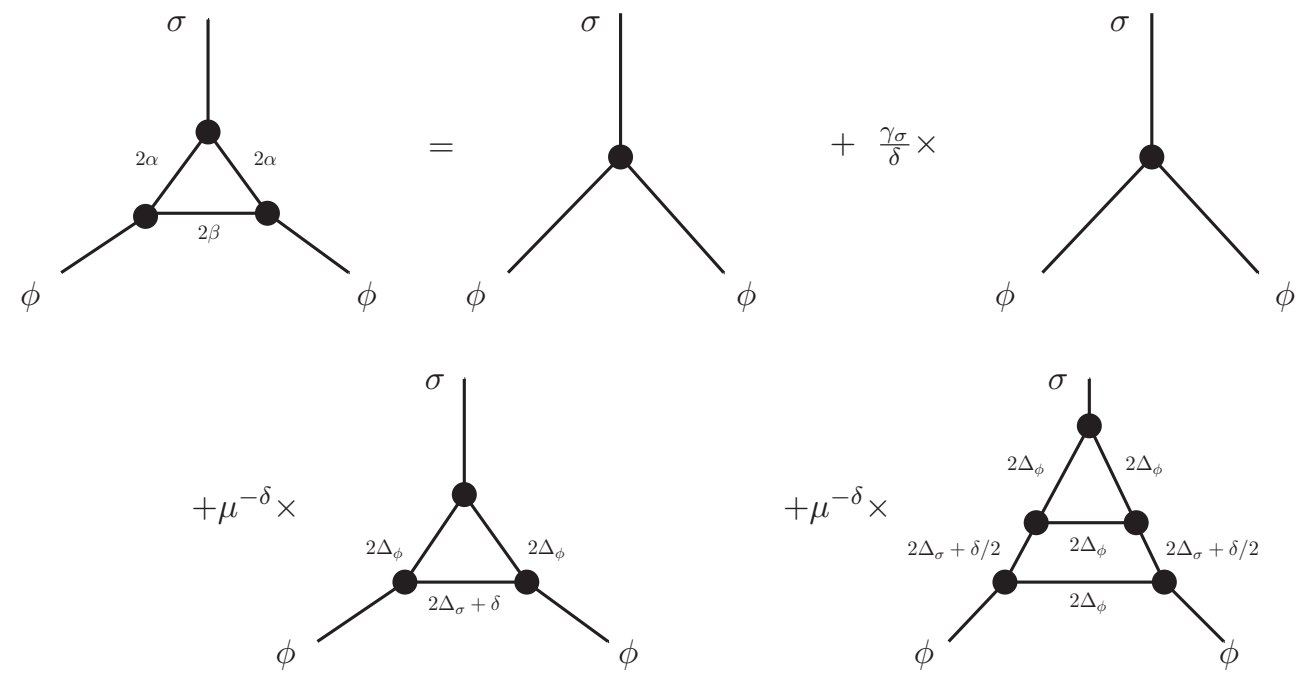
The first term in the r.h.s. of (5.6) represents the tree-level contribution of the leading-order $\phi^{2} \sigma$ interaction vertex, while the second term stands for the counter-term contribution due to the wave-function renormalization of the field $\sigma$. The last two terms in the r.h.s. of (5.6) originate due to loop corrections to the $\phi^{2} \sigma$ vertex at the next-to-leading order in $1 / N$. In the latter diagrams, we have adjusted the scaling dimension of the corresponding graphs by a small shift $\delta$ by regularizing the internal $\sigma$ lines. At the end of the calculation we will take the limit $\delta \rightarrow 0$. We will also observe explicitly that the total $1 / \delta$ pole of the second and third diagrams is cancelled out precisely by the second (counter-term) diagram. Inversely, imposing a cancellation of divergencies reproduces the correct value for the anomalous dimension $\gamma_{\sigma}$ given by (3.15).

The $\phi \phi \sigma$ conformal triangle can be used directly to calculate correlation functions and extract CFT data. For instance, in the previous paragraph, we outlined how the anomalous dimension $\gamma_{\sigma}$ can be found from such a calculation. Importantly, OPE coefficients can be calculated as well. To this end, one needs to attach to the conformal triangle the full (dressed) propagators, and integrate over the internal unique vertices: such a procedure is a direct generalization of using the Feynman rule for a tree-level vertex and integrating over an insertion of the vertex. In particular, to calculate the $\langle\phi \phi \sigma\rangle$ three-point function we proceed as follows:

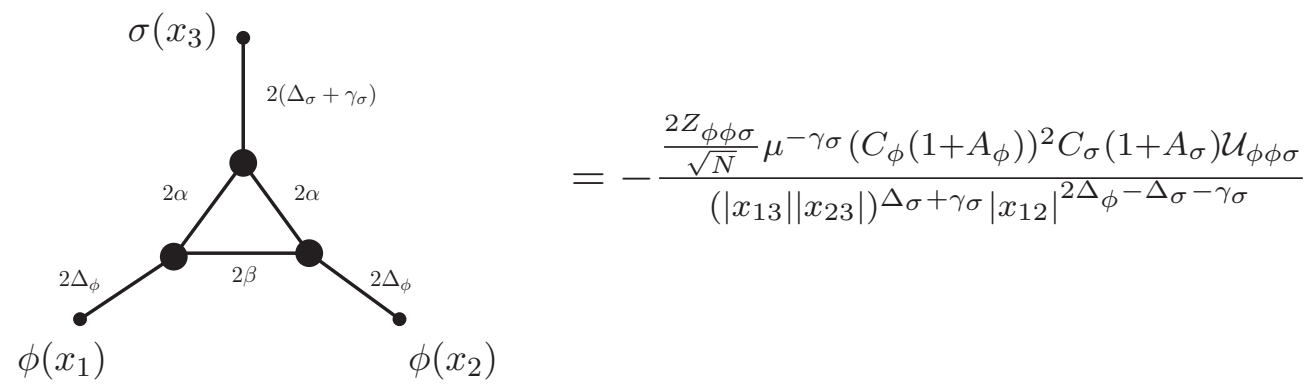

where we denoted the factor obtained from integration over three unique vertices of the conformal triangle as

$$
\begin{aligned}
\mathcal{U}_{\phi \phi \sigma}= & U\left(\frac{d-s-\gamma_{\sigma}}{2}, \frac{d-s-\gamma_{\sigma}}{2}, s+\gamma_{\sigma}\right) U\left(\frac{d-s}{2}, \frac{d-\gamma_{\sigma}}{2}, \frac{s+\gamma_{\sigma}}{2}\right) \\
& \times U\left(\frac{d-s}{2}, \frac{d-s-\gamma_{\sigma}}{2}, s+\frac{\gamma_{\sigma}}{2}\right) .
\end{aligned}
$$

Expanding (5.7) in $1 / N$ to the next-to-leading order, and comparing the result with (5.1) we obtain

$$
\begin{gathered}
\bar{C}_{\phi \phi \sigma}^{(0)}=-\frac{2 Z_{\phi \phi \sigma}^{(0)}}{\sqrt{N}} C_{\phi} C_{\sigma}^{\frac{1}{2}} \mathcal{U}_{\phi \phi \sigma}^{(0)}, \\
\delta \bar{C}_{\phi \phi \sigma}^{(0)}=\delta Z_{\phi \phi \sigma}+\delta \mathcal{U}_{\phi \phi \sigma}+A_{\phi}+\frac{A_{\sigma}}{2} .
\end{gathered}
$$


where we denoted the $1 / N$ expansion of (5.8) as

$$
\begin{aligned}
\mathcal{U}_{\phi \phi \sigma} & =\mathcal{U}_{\phi \phi \sigma}^{(0)}\left(1+\delta \mathcal{U}_{\phi \phi \sigma}\right) \\
\mathcal{U}_{\phi \phi \sigma}^{(0)} & =\frac{2 \pi^{\frac{3 d}{2}} \Gamma\left(\frac{s}{2}\right)^{4} \Gamma\left(\frac{d}{2}-s\right)^{2}}{\Gamma\left(\frac{d}{2}\right) \Gamma(s)^{2} \Gamma\left(\frac{d-s}{2}\right)^{4} \gamma_{\sigma}} \\
\delta \mathcal{U}_{\phi \phi \sigma} & =\frac{\gamma_{\sigma}}{2}\left(2 \psi^{(0)}\left(\frac{d-s}{2}\right)-3 \psi^{(0)}\left(\frac{d}{2}-s\right)+\psi^{(0)}\left(\frac{d}{2}\right)+2 \psi^{(0)}\left(\frac{s}{2}\right)-3 \psi^{(0)}(s)-\gamma\right) .
\end{aligned}
$$

Using (5.3), (5.9) we can solve for the leading-order amplitude of the conformal triangle

$$
Z_{\phi \phi \sigma}^{(0)}=\frac{U\left(\Delta_{\phi}, \Delta_{\phi}, \Delta_{\sigma}\right)}{\mathcal{U}_{\phi \phi \sigma}^{(0)}}=\frac{\Gamma\left(\frac{d}{2}\right) \Gamma(s) \Gamma\left(\frac{d-s}{2}\right)^{2} \gamma_{\sigma}}{2 \pi^{d} \Gamma\left(\frac{s}{2}\right)^{2} \Gamma\left(\frac{d}{2}-s\right)} .
$$

At the same time, (5.10) gives a prescription to calculate the $1 / N$ correction to the OPE coefficient. To finish that calculation we need to determine first the next-to-leading correction $\delta Z_{\phi \phi \sigma}$ to the $\phi \phi \sigma$ conformal triangle.

In [32] it was proposed to use the background field method to calculate conformal triangles. Such a method can be applied for the purpose of determining the values of $Z_{\phi \phi \sigma}^{(0)}$, $\delta Z_{\phi \phi \sigma}$ in our case as well. Following [32], we set the field $\sigma$ to a non-dynamical background value $\sigma \equiv \bar{\sigma}$, and attach the full $\phi$ propagators to each term on both sides of (5.6). The resulting diagrammatic equation for the propagator $\left.\langle\phi \phi\rangle\right|_{\bar{\sigma}}$ in the $\bar{\sigma}$ background is given by

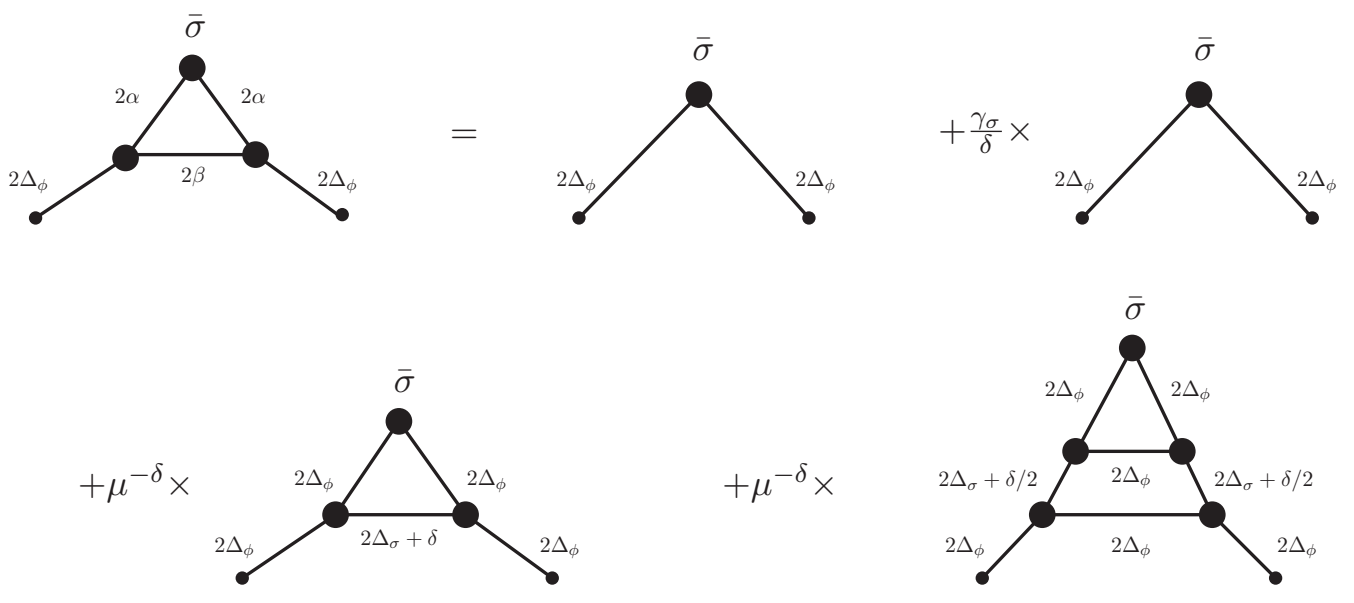

Every diagram in (5.13) can be readily calculated simply by applying the propagator merging relation, while taking the integrals starting from the topmost vertex. In particular, the 1.h.s. of (5.13) gives

$$
\text { l.h.s. } \quad \text { of } \quad(5.13)=-\frac{2}{\sqrt{N}} Z_{\phi \phi \sigma}\left(C_{\phi}\left(1+A_{\phi}\right)\right)^{2} \mathcal{U}_{\phi \phi \sigma} \frac{\bar{\sigma} \mu^{\gamma_{\sigma}}}{\left|x_{12}\right|^{d-2 s-\gamma_{\sigma}}},
$$

while we denote the four terms contributing on the r.h.s. of (5.13) as

$$
\text { r.h.s. of }(5.13)=v_{0}+v_{\text {c.t. }}+v_{1}+v_{2} \text {. }
$$


Here the tree level diagram and the vertex counter-term contribute

$$
\begin{aligned}
v_{0} & =-\frac{2}{\sqrt{N}}\left(C_{\phi}\left(1+A_{\phi}\right)\right)^{2} U\left(\frac{d-s}{2}, \frac{d-s}{2}, s\right) \frac{\bar{\sigma}}{\left|x_{12}\right|^{d-2 s}}, \\
v_{\text {c.t. }} & =-\frac{2}{\sqrt{N}}\left(C_{\phi}\left(1+A_{\phi}\right)\right)^{2} U\left(\frac{d-s}{2}, \frac{d-s}{2}, s\right) \frac{\gamma_{\sigma}}{\delta} \frac{\bar{\sigma} \mu^{\gamma_{\sigma}}}{\left|x_{12}\right|^{d-2 s-\gamma_{\sigma}}} .
\end{aligned}
$$

At the same time, for the vertex correction diagrams in the second line of (5.13) we obtain

$$
\begin{aligned}
v_{1}= & \left(-\frac{2}{\sqrt{N}}\right)^{3} C_{\phi}^{4} C_{\sigma}\left(1+A_{\phi}\right)^{2} U\left(\frac{d-s}{2}, \frac{d-s}{2}, s\right) U\left(\frac{d-s}{2}, \frac{d+\delta}{2}, \frac{s-\delta}{2}\right) \\
& \times U\left(\frac{d-s}{2}, \frac{d-s+\delta}{2}, s-\frac{\delta}{2}\right) \frac{\bar{\sigma} \mu^{\gamma_{\sigma}}}{\left|x_{12}\right|^{d-2 s+\delta}}, \\
v_{2}= & 4 C_{\phi}^{2} C_{\sigma} U\left(d-\frac{3 s}{2}, s+\frac{\delta}{4}, \frac{s}{2}-\frac{\delta}{4}\right) U\left(s+\frac{\delta}{4}, \frac{d-s}{2}+\frac{\delta}{4}, \frac{d-s-\delta}{2}\right) v_{1} .
\end{aligned}
$$

Comparing (5.14), (5.15) we first of all cancel the common factor of $-\frac{2}{\sqrt{N}}\left(C_{\phi}\left(1+A_{\phi}\right)\right)^{2} \bar{\sigma}$. Matching the leading order terms, we reproduce $Z_{\phi \phi \sigma}^{(0)}$ given by (5.12). Expanding the sub-leading contributions (5.18), (5.19) around $\delta=0$ we observe that the $1 / \delta$ poles are exactly cancelled out by the counter-term (5.17) for $\gamma_{\sigma}$ given by (3.15). At the same time, the anomalous dimension $\log \left|x_{12}\right|$ term in (5.18), (5.19) matches its counterpart on the l.h.s. (5.14) of the conformal triangle equation. Finally, matching the finite terms on both sides of this equation, we obtain the next-to-leading order correction to the conformal triangle amplitude

$$
\begin{aligned}
\delta Z_{\phi \phi \sigma}= & -\frac{1}{N} \frac{4 \Gamma\left(\frac{s}{2}\right)^{2} \Gamma(d-s)}{\Gamma\left(\frac{d}{2}\right) \Gamma(s) \Gamma\left(d-\frac{3 s}{2}\right) \Gamma\left(\frac{d-s}{2}\right)^{3} \Gamma\left(s-\frac{d}{2}\right)^{2}} \\
& \times\left(\Gamma(s) \Gamma\left(d-\frac{3 s}{2}\right) \Gamma\left(\frac{d-s}{2}\right) \Gamma\left(s-\frac{d}{2}\right)-3 \Gamma\left(\frac{s}{2}\right) \Gamma\left(\frac{d}{2}-s\right) \Gamma(d-s) \Gamma\left(\frac{3 s}{2}-\frac{d}{2}\right)\right) \\
& \times\left(-\psi^{(0)}\left(\frac{d-s}{2}\right)+\psi^{(0)}\left(\frac{d}{2}-s\right)-\psi^{(0)}\left(\frac{s}{2}\right)+\psi^{(0)}(s)\right)+\mathcal{O}\left(\frac{1}{N^{2}}\right) .
\end{aligned}
$$

With all the ingredients in place, we can calculate the $1 / N$ correction to the amplitude of the $\langle\phi \phi \sigma\rangle$ three-point function (5.10). Our result for $\delta C_{\phi \phi \sigma}$ satisfies several consistency checks. When $s=\frac{d}{2}$ the long-range fixed point becomes free, and consequently $\left.\delta C_{\phi \phi \sigma}\right|_{s=d / 2}=0$, as can be established by substituting $\sigma \sim \phi^{2}$ and performing Wick contractions. This agrees with the $s \rightarrow d / 2$ limit of (5.10).

When $s=1$, and $2<d<4$, the UV fixed point of the long-range $O(N)$ vector model was argued in [18] to be critically equivalent to the IR fixed point of an interacting 'mixed $\sigma \phi$ theory' with local kinetic terms for $\phi$ and $\sigma$, cubic interaction $\phi^{2} \sigma$ and quartic interaction $\sigma^{4}$. The critical coupling in the latter model, calculated at the leading order in the $\epsilon$-expansion performed around $d=4$ dimensions, gives [18]

$$
g_{1}^{\star}=8 \pi \sqrt{\frac{2 \epsilon}{N-32}}+\mathcal{O}\left(\epsilon^{3 / 2}\right) .
$$


This implies that in the 'mixed $\sigma \phi$ ' theory,

$$
\left.\delta \bar{C}_{\phi \phi \sigma}\right|_{s=1, d=4-\epsilon}=\frac{16}{N}+\mathcal{O}\left(\epsilon^{3 / 2}, 1 / N^{2}\right),
$$

which agrees with (5.10) for $s=1$ expanded in $d=4-\epsilon$ dimensions.

\section{$5.2\langle\sigma \sigma \sigma\rangle$}

In this section we will calculate the $\langle\sigma \sigma \sigma\rangle$ three-point function at the leading order in the $1 / N$ expansion. Expanding the trace $\log$ in the large- $N$ effective action for $\sigma(2.11)$ to $\mathcal{O}\left(1 / N^{3 / 2}\right)$ we obtain the following cubic term

$$
S_{\text {eff }} \supset-\frac{1}{3 !}\left(-\frac{2}{\sqrt{N}}\right)^{3} C_{\phi}^{3} \int d^{d} x_{1,2,3} \frac{\sigma\left(x_{1}\right) \sigma\left(x_{2}\right) \sigma\left(x_{3}\right)}{\left(\left|x_{12}\right| \mid x_{13} \| x_{23}\right)^{d-s}} .
$$

Such a non-local cubic interaction vertex can be represented diagrammatically using the $\sigma \sigma \sigma$ conformal-triangle. The corresponding Feynman rule is then given by

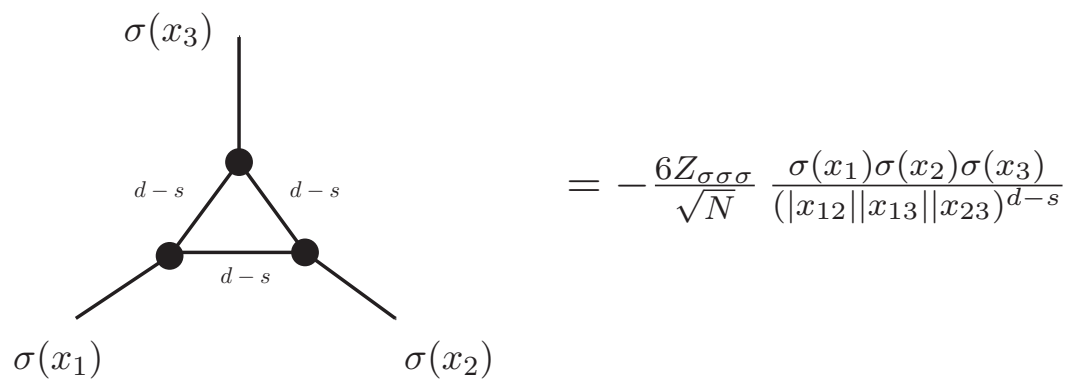

Here the leading order amplitude of the conformal triangle is given by

$$
Z_{\sigma \sigma \sigma}^{(0)}=\frac{4 C_{\phi}^{3}}{3}
$$

Attaching $\sigma$ propagators to the conformal triangle and integrating over three unique vertices we obtain the three-point function for normalized $\sigma$,

$$
\left.\left\langle\sigma\left(x_{1}\right) \sigma\left(x_{2}\right) \sigma\left(x_{3}\right)\right\rangle\right|_{\text {normalized }}=\frac{\bar{C}_{\sigma \sigma \sigma}^{(0)}}{\left(\left|x_{12}\right|\left|x_{13}\right|\left|x_{23}\right|\right)^{s}},
$$

where we denoted the normalized amplitude as

$$
\begin{aligned}
\bar{C}_{\sigma \sigma \sigma}^{(0)} & =-\frac{8}{\sqrt{N}} C_{\phi}^{3} C_{\sigma}^{\frac{3}{2}} U\left(\frac{d-s}{2}, \frac{d-s}{2}, s\right)^{2} U\left(s, \frac{s}{2}, d-\frac{3 s}{2}\right) \\
& =\frac{2^{d-2 s}(d-2 s) \sin \left(\frac{1}{2} \pi(d-2 s)\right) \Gamma\left(\frac{d}{2}-s\right)^{2} \Gamma\left(\frac{1}{2}(d-s+1)\right) \Gamma\left(\frac{3 s}{2}-\frac{d}{2}\right)}{\pi \Gamma\left(\frac{s+1}{2}\right) \Gamma\left(d-\frac{3 s}{2}\right)} \hat{C}_{\phi \phi \sigma}^{(0)} .
\end{aligned}
$$

A simple consistency check of this result is $\left.\bar{C}_{\sigma \sigma \sigma}^{(0)}\right|_{s=1, d=4-\epsilon}=0+\mathcal{O}\left(\epsilon^{\frac{3}{2}}\right)$, in agreement with the behavior of its counterpart in the dual model [18]. 


\section{Continuity of CFT data across long-range-short-range crossover}

In previous sections we derived various CFT data for the long-range conformal fixed point for a general exponent $s$ and space-time dimension $d$. At the same time, we know that when $s=2$ the long-range CFT is classically equivalent to the short-range CFT; this statement is evident by matching the actions of these two models (for instance, in momentum space). Therefore one might wonder if taking the limit $s \rightarrow 2$ in the expressions for the long-range CFT data obtained above, one would recover the known CFT data of the short-range $O(N)$ vector model. As reviewed in Introduction, it is well known that such a naive check would not fulfill that expectation; and the crossover between short-range and long-range CFTs in fact happens at the value $s=s_{\star}<2[14,15]$. Here $s_{\star}=2-2 \gamma_{\hat{\phi}}$, where $\gamma_{\hat{\phi}}$ is the anomalous dimension of $\hat{\phi}$ in the short-range model. The value of $s_{\star}$ is defined by requiring a continuity of CFT data across the long-range to the short-range transition. ${ }^{19}$ In this subsection, we provide explicit evidence for the existence of a continuous transition of all CFT data obtained above using the $1 / N$ expansion, ${ }^{20}$ namely the scaling dimensions of $\sigma$, $\sigma^{2}, \sigma \phi$, and the OPE coefficients $C_{\phi \phi \sigma}$, and $C_{\sigma \sigma \sigma}$, at the crossover point $s=s_{\star}$. This is a non-trivial consistency check of our long-range CFT data computed near a strongly-coupled point $s=s_{\star}$ in general $d$, and at the next-to-leading order in $1 / N$.

\subsection{Continuity of dimensions of $\sigma, \sigma^{2}$, and $\sigma \phi$}

Before proceeding onto the calculations in this section, we make the following simple observations regarding the leading order behaviour of the scaling dimensions and amplitudes of $\phi$ and $\sigma$,

$$
\begin{array}{rlrl}
\left.\Delta_{\phi}\right|_{s \rightarrow 2} & =\Delta_{\hat{\phi}}=\frac{d}{2}-1, & \left.\Delta_{\sigma}\right|_{s \rightarrow 2}=\Delta_{\hat{\sigma}} & =2, \\
\left.C_{\phi}\right|_{s \rightarrow 2}=C_{\hat{\phi}}=\frac{\Gamma\left(\frac{d}{2}-1\right)}{4 \pi^{\frac{d}{2}}}, & \left.C_{\sigma}\right|_{s \rightarrow 2}=C_{\hat{\sigma}}=\frac{2^{d} \Gamma\left(\frac{d-1}{2}\right) \sin \left(\frac{\pi d}{2}\right)}{\pi^{\frac{3}{2}} \Gamma\left(\frac{d}{2}-2\right)},
\end{array}
$$

To illustrate the continuity of scaling dimension of the Hubbard-Stratonovich field $\sigma$ across the crossover point $s_{\star}$ we will utilize the derivation of the anomalous dimension $\gamma_{\sigma}$ reviewed in section 3.2. The total scaling dimension of $\sigma$ is given by $\Delta_{\sigma}+\gamma_{\sigma}$, where $\Delta_{\sigma}=s$. Notice that at the crossover point $s_{\star}=2-2 \gamma_{\hat{\phi}}, \Delta_{\sigma}$ gets split into the sum of two parts, $\Delta_{\hat{\sigma}}=2$, that equals to the leading order contribution to scaling dimension of $\hat{\sigma}$, and the sub-leading term $-2 \gamma_{\hat{\phi}}$. For future purposes, let us write down this rearrangement of total scaling dimension of $\sigma$ in the long-range CFT at $s_{\star}$ as

$$
\left.\left(\Delta_{\sigma}+\gamma_{\sigma}\right)\right|_{s \rightarrow s_{\star}}=\Delta_{\hat{\sigma}}+\left(\left.\gamma_{\sigma}\right|_{s \rightarrow s_{\star}}-2 \gamma_{\hat{\phi}}\right) .
$$

Notice that terms in brackets in r.h.s. of (6.2) are sub-leading in $1 / N$, and therefore taking the limit $s \rightarrow s_{\star}=2+\mathcal{O}(1 / N)$ in those terms (more precisely, in $\gamma_{\sigma}$, since $\gamma_{\hat{\phi}}$ does not

\footnotetext{
${ }^{19}$ Note that the definition of the crossover point is independent of the value of $N$ (while the value of $s_{\star}$ is dependent on $N$ ), and can be easily generalized to the $O(N)$ model.

${ }^{20}$ Analogous calculation in the perturbative $\epsilon$-expansion, for $\epsilon=2 s-d$, was first carried out in [14] to order $\epsilon^{2}$.
} 
depend on $s$ ) needs to be replaced with taking the limit $s \rightarrow 2$. In order for the scaling dimension of the $\sigma$ field to be continuous at the crossover point $s_{\star}$,

$$
\left.\left(\Delta_{\sigma}+\gamma_{\sigma}\right)\right|_{s \rightarrow s_{\star}}=\Delta_{\hat{\sigma}}+\gamma_{\hat{\sigma}}
$$

it is then required that

$$
\left.\gamma_{\sigma}\right|_{s \rightarrow 2}=\gamma_{\hat{\sigma}}+2 \gamma_{\hat{\phi}} .
$$

To verify that the above expression is true, we notice that among the three diagrams contributing to $\langle\sigma \sigma\rangle$ at the next-to-leading order in $1 / N$ (discussed in section 3.2), two of them, namely $C_{\sigma \sigma}^{(1,2)}$, reproduce the corresponding diagrams in the short-range critical vector model when $s \rightarrow 2$, i.e. $\left.C_{\sigma \sigma}^{(1,2)}\right|_{s \rightarrow 2}=C_{\hat{\sigma} \hat{\sigma}}^{(1,2)}$. However, the third diagram, $C_{\sigma \sigma}^{(3)}$, does not reduce to its short-range counterpart $C_{\hat{\sigma} \hat{\sigma}}^{(3)}$ in that limit. Its contribution to $\gamma_{\sigma}$ is in fact absent, unlike the contribution of $C_{\hat{\sigma} \hat{\sigma}}^{(3)}$ to $\gamma_{\hat{\sigma}}$. To find the latter, we consider $C_{\hat{\sigma} \hat{\sigma}}^{(3)}$ diagram explicitly: ${ }^{21}$

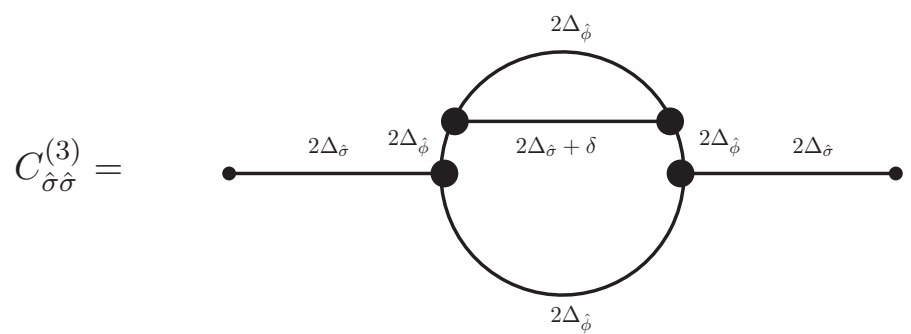

As pointed out above, this diagram is divergent in the short-range model, but its counterpart in the long-range model is finite. Importantly, the divergent behavior of this graph can be traced back to its sub-diagram, representing the $1 / N$ correction to the propagator of the field $\hat{\phi}$. However, as shown in section 3.1, the one-loop correction to the propagator of $\phi$ is finite in the long-range CFT, consistently with the expectation that scaling dimension of the field $\phi$ does not receive anomalous contributions.

We regularize $C_{\hat{\sigma} \hat{\sigma}}^{(3)}$ by adding a small shift $\delta$ to the exponent of the internal line of the Hubbard-Stratonovich field $\hat{\sigma}$, obtaining

$$
\begin{aligned}
C_{\hat{\sigma} \hat{\sigma}}^{(3)} & =\frac{C_{\hat{\sigma}} \mu^{-\delta}}{|x|^{4+\delta}} 4 C_{\hat{\phi}}^{2} C_{\hat{\sigma}}\left(\frac{2 \gamma_{\hat{\phi}}}{\delta}+A_{\hat{\phi}}\right) U\left(2, d-2+\frac{\delta}{2},-\frac{\delta}{2}\right) U\left(2, \frac{d+\delta}{2}, \frac{d-\delta}{2}-2\right) \frac{1}{(\mu|x|)^{\delta}} \\
& =\frac{C_{\hat{\sigma}}}{|x|^{4}}\left(4 \gamma_{\hat{\phi}} \log (\mu|x|)+\ldots\right),
\end{aligned}
$$

where in the last line we took the limit $\delta \rightarrow 0$ and omitted everything except for the contribution to the anomalous dimension. Consequently $C_{\hat{\sigma} \hat{\sigma}}^{(3)}$ contributes $-2 \gamma_{\hat{\phi}}$ to $\gamma_{\hat{\sigma}}$. This contribution is precisely offset by the second term in the r.h.s. of (6.4), consistent with the fact that in the long-range CFT the counterpart diagram $C_{\sigma \sigma}^{(3)}$ is finite, and therefore does not provide any contributions to $\gamma_{\sigma}$.

Remarkably, our argument for continuity of scaling dimension of $\sigma$ does not use the specific value of $\gamma_{\hat{\phi}}$. More importantly, this calculation pin-points how the continuity of the

\footnotetext{
${ }^{21}$ See [26] for a recent detailed calculation of anomalous dimension of the Hubbard-Stratonovich field.
} 
scaling dimension of $\sigma$ is inherited from continuity of the scaling dimension of $\phi$. Such an inheritance makes sense, since continuity of $\phi$ is intrinsically connected with the irrelevance of the bi-local kinetic term for $\phi$ above the crossover point.

Having established continuity of $\gamma_{\sigma}$, it is straightforward to generalize our argument to demonstrate the continuity of $\gamma_{\sigma^{2}}$. We derived the latter in section 4.1. The total dimension of $\sigma^{2}$ is given by $2 s+\gamma_{\sigma^{2}}$, which at the crossover point $s_{\star}=2-2 \gamma_{\hat{\phi}}$ can be rewritten as $4+\left(\left.\gamma_{\sigma^{2}}\right|_{s \rightarrow 2}-4 \gamma_{\hat{\phi}}\right)$. Continuity at $s_{\star}$ requires this value to be equal to $4+\gamma_{\hat{\sigma}^{2}}$, the scaling dimension of $\hat{\sigma}^{2}$ in the short-range vector model. In other words,

$$
\left.\gamma_{\sigma^{2}}\right|_{s \rightarrow 2}=\gamma_{\hat{\sigma}^{2}}+4 \gamma_{\hat{\phi}}
$$

One can see that almost all of the diagrams contributing to $\gamma_{\sigma^{2}}$ have a smooth limit $s \rightarrow 2$, under which they reduce to their short-range counterparts. The only non-trivial diagram in this limit is the one with two $\langle\sigma \sigma\rangle$ sub-diagrams, where each of these receive $1 / N$ corrections in the $s \rightarrow 2$ limit. Then (6.6) immediately follows from (6.4). It should be noted that since the anomalous dimensions of all operators $\sigma^{n}$ with $n>2$, come from the corrections to the $\sigma$ and $\sigma^{2}$ sub-diagrams, the continuity of their scaling dimensions across the crossover point follows from the continuity of the scaling dimensions of $\sigma$ and $\sigma^{2}$.

To close this subsection, we will discuss continuity of scaling dimension of the composite operator $\sigma \phi$. Scaling dimension of this composite operator was discussed in section 4.4, where we provided a quick argument due to e.o.m. for the exact value of the anomalous dimension $\gamma_{\sigma \phi}=0$ to all orders in $1 / N$, as well as an explicit perturbative calculation at the next-to-leading order in $1 / N$ expansion. Analogous calculation in the short-range vector model proceeds along the similar steps, with setting $s \rightarrow 2$ in all the sub-leading diagrams. The only adjustment which one needs to make to the long-range derivation is that the short-range $\hat{\phi}$ acquires anomalous dimension $\gamma_{\hat{\phi}}$ (the second and the third diagram in section 4.4 contributing $\gamma_{\sigma \phi}^{(2,3)}$ have a continuous behavior at $\left.s=2\right)$. Therefore the shortrange counterpart of the first diagram in section 4.4 scales as

$$
\begin{aligned}
2\left(\Delta_{\hat{\sigma}}+\gamma_{\hat{\sigma}}\right)+2\left(\Delta_{\hat{\phi}}+\gamma_{\hat{\phi}}\right) & =2\left(\left.\Delta_{\sigma}\right|_{s \rightarrow 2}+\left.\gamma_{\sigma}\right|_{s \rightarrow 2}-2 \gamma_{\hat{\phi}}\right)+2\left(\left.\Delta_{\phi}\right|_{s \rightarrow 2}+\gamma_{\hat{\phi}}\right) \\
& =\left.\left[2\left(\Delta_{\sigma}+\gamma_{\sigma}\right)+2 \Delta_{\phi}\right]\right|_{s \rightarrow s_{\star}}
\end{aligned}
$$

where we used (6.4), consistently with the continuity at $s=s_{\star}=2-2 \gamma_{\hat{\phi}}$.

\subsection{Continuity of $\langle\phi \phi \sigma\rangle$ and $\langle\sigma \sigma \sigma\rangle$}

We now proceed to demonstrate continuity of normalized three-point functions $\langle\phi \phi \sigma\rangle$, $\langle\sigma \sigma \sigma\rangle$ at the crossover point $s_{\star}$. Particularly, we are going to demonstrate the continuity of the three-point function amplitude (OPE coefficient) $\bar{C}_{\phi \phi \sigma}$ at the next-to-leading order in $1 / N$ expansion, as well as the amplitude $\bar{C}_{\sigma \sigma \sigma}$ at the leading order in $1 / N$ expansion.

Recall that we split the amplitude into the leading large- $N$ factor and the relative $1 / N$ corrections as follows:

$$
\bar{C}_{\phi \phi \sigma}=\bar{C}_{\phi \phi \sigma}^{(0)}\left(1+\delta \bar{C}_{\phi \phi \sigma}\right)
$$


Here the leading order amplitude $\bar{C}_{\phi \phi \sigma}^{(0)}$ originates from the tree-level digram, while the relative sub-leading terms $\delta \bar{C}_{\phi \phi \sigma}$ are due to next-to-leading order corrections to the $\phi \phi \sigma$ vertex. The continuity requirement is then

$$
\begin{gathered}
\bar{C}_{\hat{\phi} \hat{\phi} \hat{\sigma}}^{(0)}=\left.\bar{C}_{\phi \phi \sigma}^{(0)}\right|_{s=2}, \\
\bar{C}_{\hat{\phi} \hat{\phi} \hat{\sigma}}^{(0)} \delta \bar{C}_{\hat{\phi} \hat{\phi} \hat{\sigma}}=\left.\left(\bar{C}_{\phi \phi \sigma}^{(0)} \delta \bar{C}_{\phi \phi \sigma}-2 \gamma_{\hat{\phi}} \frac{\partial \bar{C}_{\phi \phi \sigma}^{(0)}}{\partial s}\right)\right|_{s=2} .
\end{gathered}
$$

Expressions for $\bar{C}_{\hat{\phi} \hat{\phi} \hat{\sigma}}^{(0)}, \delta \bar{C}_{\hat{\phi} \hat{\phi} \hat{\sigma}}$ are well-known [36] and therefore the continuity (6.9) can be readily checked. Using our result (5.3), (5.10) we confirm that (6.9) is indeed satisfied.

Notice that while the first relation in (6.9) is a simple consequence of (6.1), the meaning behind the second line in (6.9) is more opaque. However it does provide an interesting relation between the leading and next-to-leading contributions to the three-point function $\langle\phi \phi \sigma\rangle$.

To close this section, we notice that at the leading order in $1 / N$, continuity of $\langle\sigma \sigma \sigma\rangle$ immediately follows from (6.9) and smooth behavior of the triangle diagram.

\section{A dual description for the long-range CFT at large $N$}

As we reviewed above, the long-range critical $O(N)$ vector model is defined for the exponent $s$ taking values in the range $d / 2<s<s_{\star}$. It crosses over to the MFT regime for $s \leq d / 2$, and to the short-range regime for $s \geq s_{\star}$ (given by critical vector model plus a decoupled generalized free field $[11,12])$. The model can be studied near the MFT transition point by setting $s=d / 2+\epsilon$. This makes the quartic interaction slightly relevant, and allows one to study the model perturbatively in $\epsilon$ expansion. No such weakly coupled description of the short-range crossover point near $s=s_{\star}$ was available ${ }^{22}$ until a completely new model was suggested in [11] for the $N=1$ case (Ising model).

The proposal was to start with the action $S_{\text {crit }}$ for the short-range critical Ising model $(N=1$ vector model), and couple it to a generalized free field $\chi$,

$$
S=S_{\text {crit }}+\int d^{d} x \int d^{d} y \frac{\chi(x) \chi(y)}{|x-y|^{d-s}}+\lambda \int d^{d} x \hat{\phi} \chi .
$$

The scaling dimension of $\chi$ is therefore fixed to be $\Delta_{\chi}=\frac{d+s}{2}$, while the field $\hat{\phi}$ (which is the lowest scalar primary of the critical Ising CFT) has dimension $\frac{d}{2}-1+\gamma_{\hat{\phi}}=\frac{d-s_{\star}}{2}$, when $\lambda=0$. The dimension of $\chi$ was tuned such that the perturbation $\hat{\phi} \chi$ is irrelevant for $s>s_{\star}$, and relevant for $s<s_{\star}$. In the latter case, the coupling $\lambda$ triggers an RG flow that can be studied perturbatively in the vicinity of $s_{\star}$. Interestingly, the IR fixed point of this flow is described by a long-range CFT. Moreover, it has been suggested in [11] that the resulting long-range CFT is, for all $d / 2 \leq s \leq s_{\star}$, the same as the long-range critical vector model, obtained at the end of the RG flow of the Gaussian MFT deformed by a quartic

\footnotetext{
${ }^{22}$ With [14] providing an earlier attempt of the construction perturbative near $s_{\star}$, as well as near $d=4$.
} 
operator. Therefore, these two UV descriptions are dual in the IR, and have complementary perturbative regimes on the opposite ends of the range $d / 2 \leq s \leq s_{\star}$. However, evidence for the duality for all intermediate values of $s$ has so far been obtained only for certain three-point function coefficient ratios, valid for all orders in the $\epsilon$-expansion [11].

In this section, we aim to provide a complete non-perturbative description of the duality for all values of $s$, by reformulating the duality suggested in [11] at large $N$. We begin with re-writing the action for the short-range critical $O(N)$ vector model at large $N$ in the Hubbard-Stratonovich formalism,

$$
S_{\text {crit }}=\int d^{d} x\left(\frac{1}{2}\left(\partial_{\mu} \hat{\phi}\right)^{2}-\frac{\hat{\sigma}^{2}}{4 g}+\frac{1}{\sqrt{N}} \hat{\sigma} \hat{\phi}^{2}\right)+\cdots,
$$

where ellipsis stands for $1 / N$ corrections, and we also skip explicitly writing the counterterms. Following [11], we introduce a new field $\chi^{i}, i=1, \ldots, N$ with a bi-local kinetic term, and scaling dimension

$$
\Delta_{\chi}=\frac{d+s}{2}
$$

and couple it to the field $\hat{\phi}^{i}$ as follows:

$$
S=S_{\text {crit }}+a \int d^{d} x \int d^{d} y \frac{\chi^{i}(x) \chi^{i}(y)}{|x-y|^{d-s}}+\lambda \int d^{d} x \hat{\phi}^{i} \chi^{i}
$$

Here, the scaling dimension of $\hat{\phi}^{i}$ is $\left(d-s_{\star}\right) / 2$. The kinetic term normalization coefficient $a$ can be arbitrary, and reflects conventions regarding the definition of $\chi$. A convenient choice to fix it will be explained momentarily. To obtain a large $N$ description of the IR CFT, we first perform the following redefinition of the fields and couplings,

$$
\Phi^{i}=\lambda \hat{\phi}^{i}, \quad \Sigma=\frac{1}{\lambda^{2}} \hat{\sigma}, \quad g=\lambda^{4} G .
$$

This allows us to re-write the action $\operatorname{as}^{23}$

$$
S_{\mathrm{SR} \chi}=a \int d^{d} x \int d^{d} y \frac{\chi(x) \chi(y)}{|x-y|^{d-s}}+\int d^{d} x\left(\frac{1}{2 \lambda^{2}}(\partial \Phi)^{2}-\frac{1}{4 G} \Sigma^{2}+\Phi \chi+\frac{1}{\sqrt{N}} \Sigma \Phi^{2}\right)+\cdots,
$$

where we have again omitted higher-order corrections to vertices and propagators.

We will now argue that when both $G$ and $\lambda$ are tuned to criticality, and $s$ assumes values in the range $d / 2<s<s_{\star}$, the model (7.6) is equivalent to the long-range CFT (2.10). The argument can be constructed by going from the latter towards the former.

Starting from the long-range CFT model (2.10), we can add to it its action a local kinetic term $\frac{1}{2 \lambda^{2}}(\partial \phi)^{2}$, where $\lambda$ is a dimensionful constant that we choose to be equal to the IR critical value of the coupling constant in the model (7.6). This can be done without affecting dynamics of the field $\phi$ in the range $d / 2<s<s_{\star}$, where such a term is suppressed compared to the bi-local kinetic term for the field $\phi$.

\footnotetext{
${ }^{23}$ We skip keeping track of the $O(N)$ indices.
} 
We then insert an identity into the partition function $Z_{\mathrm{LR}}$ of the model (2.10), represented as a Gaussian integral over a field $\chi{ }^{24}$

$$
\begin{aligned}
Z_{\mathrm{LR}} \rightarrow & Z_{\mathrm{LR}} \frac{1}{z} \int[D \chi] \exp \left(-a \int \frac{d^{d} x_{1,2}}{\left|x_{12}\right|^{d-s}}\left(\chi+\alpha \int d^{d} x_{1}^{\prime} \frac{\phi\left(x_{1}^{\prime}\right)}{\left|x_{1}-x_{1}^{\prime}\right|^{d+s}}\right)\right. \\
& \left.\times\left(\chi+\alpha \int d^{d} x_{2}^{\prime} \frac{\phi\left(x_{2}^{\prime}\right)}{\left|x_{2}-x_{2}^{\prime}\right|^{\mid d+s}}\right)\right)
\end{aligned}
$$

accompanied by the corresponding normalization prefactor $1 / z$. Choosing

$$
\begin{aligned}
a & =-\frac{1}{4 C(s) \pi^{d} A\left(\Delta_{\Phi}\right) A\left(d-\Delta_{\Phi}\right)}, \\
\alpha & =\frac{1}{2 a \pi^{d} A\left(\Delta_{\Phi}\right) A\left(d-\Delta_{\Phi}\right)}=-2 C(s),
\end{aligned}
$$

we obtain that the action (2.10) transforms into

$$
S_{\mathrm{LR}}=a \int d^{d} x \int d^{d} y \frac{\chi(x) \chi(y)}{|x-y|^{d-s}}+\int d^{d} x\left(\frac{1}{2 \lambda^{2}}(\partial \phi)^{2}-\frac{1}{4 g} \sigma^{2}+\phi \chi+\frac{1}{\sqrt{N}} \sigma \phi^{2}\right) .
$$

Under the identification

$$
\Phi \leftrightarrow \phi, \quad \Sigma \leftrightarrow \sigma, \quad g \leftrightarrow G
$$

the models (7.6), (7.10) are indeed equivalent.

Notice that when $s>s_{\star}$ the coupling $\lambda$ in (7.4) becomes irrelevant, and its critical value is a trivial $\lambda=0$, making the field redefinition (7.5) ill-defined. In fact, the field $\chi$ decouples into an independent d.o.f. with a bi-local kinetic term. Therefore while the IR duality between the long-range vector model and the deformed short-range critical vector model, defined for $d / 2<s<s_{\star}$, is quite straightfoward to establish, a more subtle prediction is that when $s>s_{\star}$ (outside the scope of the long-range model, and therefore beyond the regime where one can talk about such a duality) the system retains a decoupled generalized free field d.o.f $[11,12] .^{25}$

Having argued for the duality between the long-range and the deformed short-range critical vector models in the range $d / 2<s<s_{\star}$, we would like to explore the meaning of the dual of the generalized free field $\chi$ on the long-range side, with an aim to make the duality more precise. Motivated by this goal, for the rest of this section we are going to return to the picture with the original field $\chi$. The resulting action of the critical regime of the $O(N)$ vector model coupled to $\chi$ is given by

$$
S=-\frac{1}{4 C(s) \pi^{d} A\left(\Delta_{\Phi}\right) A\left(d-\Delta_{\Phi}\right)} \int d^{d} x \int d^{d} y \frac{\chi(x) \chi(y)}{|x-y|^{d-s}}+\int d^{d} x\left(\Phi \chi+\frac{1}{\sqrt{N}} \Sigma \Phi^{2}\right) .
$$

\footnotetext{
${ }^{24}$ Our notation for this field is deliberately the same as for the $\chi$ field in the action (7.6), as we intend to identify these d.o.f. on both sides of the argued IR duality.

${ }^{25}$ The dual description of the critical long-range model is also useful near $s=s_{\star}$, where it gives a perturbative handle on the calculations $[11,12]$.
} 
It follows from the action (7.12) that the propagator of the field $\chi$ is

$$
\langle\chi(x) \chi(0)\rangle=\frac{C_{\chi}\left(1+A_{\chi}\right)}{|x|^{2 \Delta_{\chi}}}, \quad C_{\chi}=-2 C(s),
$$

where we took into account that the dimension $\Delta_{\chi}$ is exact, and the only possible $1 / N$ corrections to the $\chi$ propagator can go into the amplitude correction $A_{\chi}$. At the same time, the $\chi$ e.o.m. following from (7.12) is,

$$
\Phi=\frac{1}{2 C(s) \pi^{d} A\left(\Delta_{\Phi}\right) A\left(d-\Delta_{\Phi}\right)} \int d^{d} y \frac{\chi(y)}{|x-y|^{d-s}},
$$

which, in turn, gives

$$
\langle\Phi(x) \Phi(0)\rangle=-\frac{1+A_{\chi}}{2 \pi^{d} C(s) A\left(\Delta_{\Phi}\right) A\left(d-\Delta_{\Phi}\right)} \frac{1}{|x|^{2 \Delta_{\Phi}}} .
$$

Important manifestation of a duality between two CFTs is given by matching conformal correlation functions on both sides of the duality. When matching amplitudes of the correlation functions of operators in different models, one needs to ensure consistent normalization of these operators. A convenient choice is given by normalization of the two-point functions of the considered operators to unity. ${ }^{26}$ Particularly, one finds it useful that rescaling the fields $\chi, \Phi$ as

$$
\chi \rightarrow \frac{1}{\sqrt{-2 C(s)\left(1+A_{\chi}\right)}} \chi, \quad \Phi \rightarrow \sqrt{-\frac{2 \pi^{d} C(s) A\left(\Delta_{\Phi}\right) A\left(d-\Delta_{\Phi}\right)}{1+A_{\chi}}} \Phi,
$$

obtaining unit-normalized propagators $\langle\chi \chi\rangle,\langle\Phi \Phi\rangle$. The three-point functions involving the fields $\chi, \Phi$ will be calculated for such fields normalized according to (7.16). Similar normalization conventions can be used for their counterparts in the long-range model.

In particular, one can establish that the ratios of coefficients of three-point functions $\left\langle\chi \mathrm{O}_{2} \mathrm{O}_{3}\right\rangle,\left\langle\Phi \mathrm{O}_{2} \mathrm{O}_{3}\right\rangle$, and $\left\langle\sigma \phi \mathrm{O}_{2} \mathrm{O}_{3}\right\rangle,\left\langle\phi \mathrm{O}_{2} \mathrm{O}_{3}\right\rangle$ where $\mathrm{O}_{2,3}$ are some conformal operators, match exactly to all orders in $1 / N$ and for all $d / 2<s<s_{\star}$, analogously to [11, 12]. This implies the following matching of d.o.f. on both sides of the duality:

$$
\chi \leftrightarrow \sigma \phi .
$$

Simply put, the relation (7.17) between $\chi$ and $\sigma \phi$ follows from the relation (7.11) $\Phi \leftrightarrow \phi$, accompanied by the observation that the pairs of fields $\sigma \phi, \phi$ and $\chi, \Phi$ satisfy analogous equations of motion (4.15), (7.14).

An infra-red duality between the critical long-range model and the short-range model deformed by a coupling to the generalized free field $\chi$, requires one to match the spectrum of the two CFTs, as well as all of the CFT data. An immediate observation one can make is that in the $N=1$ case the long-range model possesses an operator $\phi^{3}$ with exact scaling dimension $(d+s) / 2$, while the short-range model has no analogous counterpart [11]. The corresponding operator in the critical long-range $O(N)$ model in the Hubbard-Stratonovich

\footnotetext{
${ }^{26}$ See $[26,34,37]$ for a recent discussion.
} 
language is $\sigma \phi$, whose dimension $\Delta_{\sigma \phi}$ given by (4.17), as we derived in section 4.4, is fixed exactly by the e.o.m. relating it to the dimension of $\phi$. Together with the other operator $\phi$ of a fixed scaling dimension (2.6), the 'shadow relation' is satisfied, analogously to the $N=1$ case of $[10]$

$$
\Delta_{\phi}+\Delta_{\sigma \phi}=d
$$

Notice that absence of the anomalous dimension contribution to the composite operator $\sigma \phi$ in the long-range critical model is to be contrasted with its counterpart $\hat{\sigma} \hat{\phi}$ in the shortrange vector model. This being said, the full scaling dimension of $\sigma \phi$ is continuous across $s_{\star}$, as we demonstrated in section 6.1.

We close this subsection by pointing out that using e.o.m. (4.15) we can also calculate the following cross-correlator

$$
\langle\sigma \phi(x) \phi(0)\rangle=-\frac{C(s)}{\sqrt{N}} C_{\phi} \pi^{d} A\left(\frac{d-s}{2}\right) A\left(\frac{d+s}{2}\right) \delta^{(d)}(x),
$$

which vanishes for non-coincident points. The counterpart of this correlator in the deformed short-range model is given by $\langle\chi(x) \hat{\phi}(0)\rangle \simeq \delta^{(d)}(x)$. Notice that the result (7.19) is exact to all orders in $1 / N$, but can be seen explicitly at the leading order in $1 / N$ from the diagram

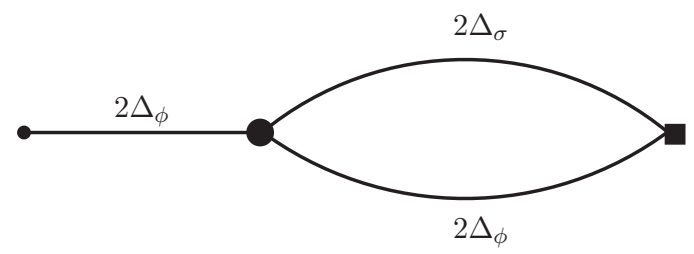

\subsection{Anomalous dimension of the stress-energy tensor}

In this section, we continue studying the $\hat{\phi} \chi$ perturbation (7.4) of the short-range $O(N)$ vector model (7.2) that brings it to a long-range critical regime in the IR. Our focus will be on the fate of the stress-energy tensor operator $T_{\mu \nu}$,

$$
T_{\mu \nu}=\partial_{\mu} \hat{\phi} \partial_{\nu} \hat{\phi}-\delta_{\mu \nu}\left(\frac{1}{2}(\partial \hat{\phi})^{2}-\frac{1}{4 g_{\star}} \hat{\sigma}^{2}+\frac{1}{\sqrt{N}} \hat{\sigma} \hat{\phi}^{2}\right)
$$

of the original short-range vector model (7.2) under such a deformation. In (7.20) we have substituted the critical value of the coupling $g_{\star}$. One anticipates that conservation of the composite operator (7.20) is broken at the long-range fixed point of the model $(7.4)$ [11, 12], where it no longer has the status of a conserved stress-energy tensor. In agreement with such an expectation, working at the first order in $1 / N$ expansion, we will derive anomalous dimension and trace of the operator (7.20).

Unlike calculation of the previous section (that was carried out in the entire range $\left.d / 2<d<s_{\star}\right)$, in this section we will stay perturbatively close to the cross-over point $s_{\star}$, working at the linear order in $\delta=\left(s_{\star}-s\right) / 2$. Since our starting point is the short-range critical vector model, we find it convenient for the purposes of this section to work in terms of the original conventions $\hat{\phi}, \hat{\sigma}$ for the degrees of freedom of this model. 
While in the original theory, $\lambda=0$, the stress-energy tensor (7.20) is conserved on shell, conservation law of that operator is broken at the new fixed point, where it no longer plays the role of stress-energy tensor. This can manifest in the anomalous dimension $\gamma_{T}$ and trace $\hat{c}$, in the ansatz for the correlation function ${ }^{27}$

$$
\left\langle\partial_{\mu} T^{\mu \nu}\left(x_{1}\right) \partial_{\lambda} T^{\lambda \rho}\left(x_{2}\right)\right\rangle=\frac{\partial}{\partial x_{1}^{\mu}} \frac{\partial}{\partial x_{2}^{\lambda}}\left(C_{T} \frac{I^{\mu \nu, \lambda \rho}\left(x_{12}\right)}{\left|x_{12}\right|^{2 d+2 \gamma_{T}}}+\hat{c} \delta^{\mu \nu} \delta^{\lambda \rho} \frac{1}{\left|x_{12}\right|^{2 d}}\right),
$$

where $\gamma_{T}$ is the anomalous dimension, $C_{T}$ is the central change of the short-range theory,

$$
C_{T}=N\left(\frac{d}{(d-1) S_{d}^{2}}+\mathcal{O}\left(\frac{1}{N}\right)\right), \quad S_{d}=\frac{2 \pi^{\frac{d}{2}}}{\Gamma\left(\frac{d}{2}\right)},
$$

We also defined tensor structures

$$
\begin{aligned}
I_{\mu \nu}(x) & =\delta_{\mu \nu}-2 \frac{x_{\mu} x_{\nu}}{x^{2}} \\
I_{\mu \nu, \lambda \rho}(x) & =\frac{1}{2}\left(I_{\mu \lambda}(x) I_{\nu \rho}(x)+I_{\mu \rho}(x) I_{\nu \lambda}(x)-\frac{2}{d} \delta_{\mu \nu} \delta_{\lambda \rho}\right),
\end{aligned}
$$

and the $\hat{c}$ term in (7.21) accounts for a possible non-vanishing trace. ${ }^{28}$ Simplifying (7.21), while linearizing in $\delta$, we obtain

$$
\left\langle\partial_{\mu} T^{\mu \nu}(x) \partial_{\lambda} T^{\lambda \rho}(0)\right\rangle=\frac{C_{T}(d-1)(d+2) \gamma_{T}}{d} I^{\nu \rho} \frac{1}{|x|^{2 d+2}}+2 d \hat{c}\left(I^{\nu \rho}-2 d \frac{x^{\nu} x^{\rho}}{|x|^{2}}\right) \frac{1}{|x|^{2 d+2}} .
$$

We are going to compare the general ansatz (7.25) with what we obtain specifically in the model (7.4). Working at the second order in conformal perturbation theory in $\lambda_{\star}$ we derive

$$
\begin{aligned}
& \left\langle\partial_{\mu} T^{\mu \nu}(x) \partial_{\lambda} T^{\lambda \rho}(0)\right\rangle=\frac{\lambda_{\star}^{2}}{2} \int d^{d} x_{1,2}\left\langle\partial_{\mu} T^{\mu \nu}(x) \partial_{\lambda} T^{\lambda \rho}(0) \hat{\phi}\left(x_{1}\right) \chi\left(x_{1}\right) \hat{\phi}\left(x_{2}\right) \chi\left(x_{2}\right)\right\rangle+\mathcal{O}\left(\lambda_{\star}^{3}\right) \\
& =\frac{\lambda_{\star}^{2}}{2} \int d^{d} x_{1,2}\left\langle\chi\left(x_{1}\right) \chi\left(x_{2}\right)\right\rangle\left\langle\partial_{\mu} T^{\mu \nu}(x) \partial_{\lambda} T^{\lambda \rho}(0) \hat{\phi}\left(x_{1}\right) \hat{\phi}\left(x_{2}\right)\right\rangle+\mathcal{O}\left(\lambda_{\star}^{3}\right) \\
& =\lambda_{\star}^{2}\langle\chi(x) \chi(0)\rangle\left\langle\partial^{\nu} \hat{\phi}(x) \partial^{\rho} \hat{\phi}(0)\right\rangle+\mathcal{O}\left(\lambda_{\star}^{3}\right),
\end{aligned}
$$

where in the last line we used Ward identity for the divergence of the stress-energy tensor in the original short-range sector of the model. From (7.26) we then obtain

$$
\left\langle\partial_{\mu} T^{\mu \nu}(x) \partial_{\lambda} T^{\lambda \rho}(0)\right\rangle=N \lambda_{\star}^{2}\left(d-s_{\star}\right) C_{\hat{\phi}} C_{\chi} \frac{1}{|x|^{2 d+2}}\left(I^{\nu \rho}-\left(d-s_{\star}\right) \frac{x^{\nu} x^{\rho}}{|x|^{2}}\right),
$$

where we substituted $s=s_{\star}$ at the short-range fixed point.

Comparing $(7.27),(7.25)$ while demanding that tensor structures match, we arrive at

$$
\begin{aligned}
\hat{c} & =N \lambda_{\star}^{2} \frac{\left(d-s_{\star}\right)^{2} C_{\hat{\phi}} C_{\chi}}{4 d^{2}}+\mathcal{O}\left(\lambda_{\star}^{3}\right), \\
\gamma_{T} & =N \lambda_{\star}^{2} \frac{\left(d+s_{\star}\right)\left(d-s_{\star}\right) C_{\hat{\phi}} C_{\chi}}{2 C_{T}(d-1)(d+2)}+\mathcal{O}\left(\lambda_{\star}^{3}\right) .
\end{aligned}
$$

\footnotetext{
${ }^{27}$ Here we skipped $2 \gamma_{T}$ in the exponent in the second term in the r.h.s., anticipating linearization in $\delta$, while taking into account that both $\gamma_{T}$ and $\hat{c}$ vanish when $\delta=0$.

${ }^{28}$ We thank M. Smolkin for discussion of this point.
} 
In the perturbative regime, near the short-range fixed point, we have [11]

$$
\lambda_{\star}^{2}=\alpha(d) \delta+\mathcal{O}\left(\delta^{2}\right),
$$

where $\delta=\left(s_{\star}-s\right) / 2$. Furthermore, plugging $s_{\star}=2-2 \gamma_{\hat{\phi}}$ in (7.28), (7.29) and expanding in $\gamma_{\hat{\phi}}$ (which in the large $N$ language means expanding in $1 / N$ ), we obtain

$$
\begin{aligned}
\hat{c} & =N \alpha \delta \frac{(d-2) \Gamma\left(\frac{d}{2}+1\right)^{2}}{d^{3} \pi^{d}} \gamma_{\hat{\phi}}+\mathcal{O}\left(\delta^{2}, \gamma_{\hat{\phi}}^{2}\right), \\
\gamma_{T} & =\frac{2 \alpha \delta}{\Gamma\left(\frac{d}{2}\right)^{2}} \gamma_{\hat{\phi}}+\mathcal{O}\left(\delta^{2}, \gamma_{\hat{\phi}}^{2}\right) .
\end{aligned}
$$

While the anomalous dimension and trace (7.31), (7.32) have been derived perturbatively close to the short-range fixed point, the operator $\partial_{\mu} T^{\mu \nu}$ can be studied in the entire range $d / 2<s<s_{\star}$. We can decompose this operator in terms of the primary [11, 38]

$$
V^{\nu}=\hat{\phi} \partial^{\nu} \chi-\frac{\Delta_{\chi}}{\Delta_{\phi}} \chi \partial^{\nu} \hat{\phi}
$$

and the descendant $\partial^{\nu}(\hat{\phi} \chi)$. Notice that the latter has a vanishing (leading order) crosscorrelator with (7.33). To find coefficients of such a decomposition, we will be working in the conformal perturbation theory at linear order in $\lambda_{\star}$ :

$$
\begin{aligned}
\left\langle\partial_{\mu} T^{\mu \nu}(x) \partial^{\rho}(\hat{\phi} \chi)(0)\right\rangle & =\lambda_{\star} \int d^{d} x_{1}\left\langle\partial_{\mu} T^{\mu \nu}(x) \partial^{\rho}(\hat{\phi} \chi)(0) \hat{\phi} \chi\left(x_{1}\right)\right\rangle \\
& =\lambda_{\star}\left\langle\partial^{\nu} \hat{\phi} \chi(x) \partial^{\rho}(\hat{\phi} \chi)(0)\right\rangle,
\end{aligned}
$$

where in the last line we used Ward identity. Similarly, we obtain

$$
\left\langle\partial_{\mu} T^{\mu \nu}(x) V^{\rho}(0)\right\rangle=\lambda_{\star}\left\langle\partial^{\nu} \hat{\phi} \chi(x) V^{\rho}(0)\right\rangle .
$$

Therefore

$$
\partial_{\mu} T^{\mu \nu}(x)=\lambda_{\star} \partial^{\nu} \hat{\phi} \chi+\mathcal{O}\left(\lambda_{\star}^{2}\right)
$$

which we can rewrite as

$$
\partial_{\mu} T^{\mu \nu}=-\lambda_{\star} \frac{d-s}{2 d} V^{\nu}-\frac{d-s}{2 d} \lambda_{\star} \partial^{\nu}(\Phi \chi) .
$$

A short-cut to the calculation of $\gamma_{T}$, providing a faster alternative derivation of the result (7.29), is afforded by the decomposition (7.37). One can easily see that focusing only on the $V^{\nu}$ contribution to the divergence of the stress-energy tensor (7.37), while simultaneously ignoring that trace contribution $\hat{c}$ in the general ansatz (7.25), reproduces the final expression (7.29) for the anomalous dimension $\gamma_{T}[11,12]{ }^{29}$ Indeed, following such a strategy we obtain from (7.37)

$$
\left\langle\partial_{\mu} T^{\mu \nu}(x) \partial_{\lambda} T^{\lambda \rho}(0)\right\rangle=\lambda_{\star}^{2} \frac{(d-s)^{2}}{4 d^{2}}\left\langle V^{\nu}(x) V^{\rho}(0)\right\rangle+\ldots .
$$

\footnotetext{
${ }^{29}$ In particular, one can see that the coefficient in front of the $V^{\nu}$ term in r.h.s. of (7.37) matches with its counterpart $b\left(g_{\star}\right)=b_{1} g_{\star}+\mathcal{O}\left(g_{\star}^{2}\right)$ introduced in [11, 12].
} 
Substituting here

$$
\left\langle V^{\nu}(x) V^{\rho}(0)\right\rangle=N \frac{2 d C_{\hat{\phi}} C_{\chi}(d+s)}{d-s} \frac{1}{|x|^{2 d+2}} I^{\nu \rho}
$$

and comparing the result with the $\gamma_{T}$ term in (7.25) we recover (7.29).

\section{Discussion}

The main focus of this paper was to study the long-range critical $O(N)$ vector model in the large- $N$ limit. This model appears in the IR regime of the Gaussian MFT for a generalized free field, deformed by a local quartic interaction. Working within the HubbardStratonovich formalism, we calculated several new scaling dimensions and OPE coefficients of various primary operators in this model, performing most of our calculations at the next-to-leading order in the $1 / N$ expansion. The CFT data we obtained furnishes a nontrivial consistency check for the existence of the full conformal symmetry at the long-range fixed point.

In particular, we determined the leading order contribution to the anomalous dimension of the composite operator $\sigma^{n}, n \geq 2$, and calculated the three-point functions $\langle\phi \phi \sigma\rangle,\langle\sigma \sigma \sigma\rangle$. We also established that the cross-correlator $\left\langle\sigma^{2} \sigma\right\rangle$ vanishes, at least at the leading order in $1 / N$, unlike its short-range counterpart discussed in $[29,30]$. The vanishing of this correlator simultaneously indicates that the long-range fixed point is indeed a CFT, and that $\sigma^{2}$ is in fact a primary operator in this CFT.

While in the short-range CFT certain composite operators, such as $\hat{\sigma}^{2}$, can mix with the descendants, such as $\partial^{2} \hat{\sigma}$, such a mixing is impossible in the long-range CFT for the Hubbard-Stratonovich field with a leading-order scaling dimension $s$, taking a general value in the range $d / 2<s<s_{\star}$ (see [13] for a bootstrap analysis of the long-range Ising model). The case of $s=1$ requires a special attention, since it allows for the possibility of operator mixing, at least in principle, such as a mixing between $\sigma^{4}$ and $\partial^{2} \sigma^{2}$. Notice that $s=1$ in fact has the physical interpretation of a boundary CFT (BCFT), i.e., a free field theory in the $d+1$-dimensional bulk perturbed by a quartic interaction localized on its $d$-dimensional boundary (or defect). ${ }^{30}$ We therefore expect the spectrum of conformal primaries to shrink in the BCFT, as contrasted to a generic long-range CFT. It would be interesting to explore this direction further.

We have also investigated the interplay between long-range and short-range critical vector models. While the former is expected to occupy the region $d / 2<s<s_{\star}$ of the exponent parameter $s$, the latter exists in the region $s>s_{\star}$. We have performed an explicit consistency check of our results for the calculated CFT data, by demonstrating its continuity at the long-range-short-range crossover point $s_{\star}$. Furthermore, generalizing the construction of $[11,12]$ to the large- $N$ case, we have argued for the existence of an exact IR duality between the long-range vector model, and the short-range model deformed by the

\footnotetext{
${ }^{30}$ For the quartic operator to be relevant in the IR one needs to consider $d<2$. When $d>2$ the theory arrives at the critical regime in the UV, however it suffers from usual instabilities typically found in such cases [18].
} 
coupling to a generalized free field. Performing a simultaneous perturbative expansion to the linear order in $s_{\star}-s$ and to the next-to-leading order in $1 / N$ we also obtained expression for the anomalous dimension and trace of the stress-energy tensor of the short-range vector model, which it acquires at the long-range fixed point.

To close this discussion, we would like to notice that long-range critical vector models has recently received attention from the perspective of persistent symmetry breaking at all temperatures [39], adding extra motivation for expanding our understanding of CFT data of these models.

\section{Acknowledgments}

We thank M. Smolkin, S. Rychkov for helpful discussions. Our work is partially supported by the Binational Science Foundation (grant No. 2016186), the Israeli Science Foundation Center of Excellence (grant No. 2289/18), and by the Quantum Universe I-CORE program of the Israel Planning and Budgeting Committee (grant No. 1937/12). The work of NC is partially supported by Yuri Milner scholarship.

\section{A Some useful identities}

In this appendix we collect some useful expressions and identities.

Loop diagram in the position space are simply additive:

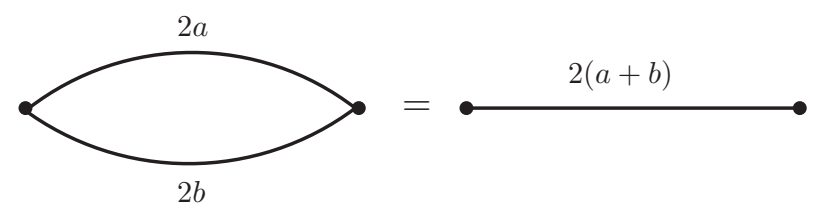

The propagator merging relation is given by

$$
\int d^{d} x_{2} \frac{1}{\left|x_{2}\right|^{2 a}\left|x_{1}-x_{2}\right|^{2 b}}=U(a, b, d-a-b) \frac{1}{\left|x_{1}\right|^{2 a+2 b-d}},
$$

where we introduced

$$
U(a, b, c)=\pi^{\frac{d}{2}} A(a) A(b) A(c)
$$

This relation can be diagrammatically represented as

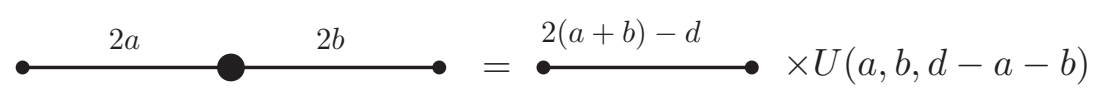

Uniqueness relation for $a_{1}+a_{2}+a_{3}=d$ is written as [40, 41]

$$
\int d^{d} x \frac{1}{\left|x_{1}-x\right|^{2 a_{1}}\left|x_{2}-x\right|^{2 a_{2}}\left|x_{3}-x\right|^{2 a_{3}}}=\frac{U\left(a_{1}, a_{2}, a_{3}\right)}{\left|x_{12}\right|^{d-2 a_{3}}\left|x_{13}\right|^{d-2 a_{2}}\left|x_{23}\right|^{d-2 a_{1}}},
$$


and is graphically represented as
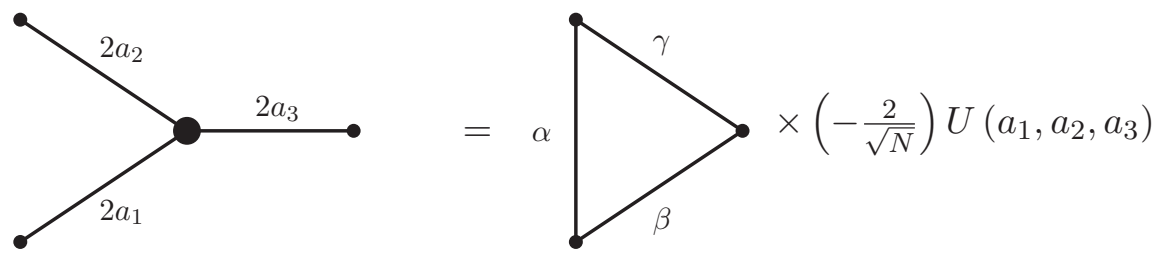

Here we defined $\alpha=d-2 a_{3}, \beta=d-2 a_{2}, \gamma=d-2 a_{1}$.

Open Access. This article is distributed under the terms of the Creative Commons Attribution License (CC-BY 4.0), which permits any use, distribution and reproduction in any medium, provided the original author(s) and source are credited.

\section{References}

[1] F.J. Dyson, Existence of a phase transition in a one-dimensional Ising ferromagnet, Commun. Math. Phys. 12 (1969) 91 [inSPIRE].

[2] M.E. Fisher, S.-k. Ma and B.G. Nickel, Critical Exponents for Long-Range Interactions, Phys. Rev. Lett. 29 (1972) 917 [InSPIRE].

[3] J.M. Kosterlitz, Phase Transitions in Long-Range Ferromagnetic Chains, Phys. Rev. Lett. 37 (1976) 1577 [INSPIRE].

[4] M. Aizenman and R. Fernandez, Critical exponents for long-range interactions, Lett. Math. Phys. 16 (1988) 39.

[5] D.C. Brydges, P.K. Mitter and B. Scoppola, Critical $\left(\Phi^{4}\right)_{3, \epsilon}$, Commun. Math. Phys. 240 (2003) 281 [hep-th/0206040] [inSPIRE].

[6] A. Abdesselam, A Complete Renormalization Group Trajectory Between Two Fixed Points, Commun. Math. Phys. 276 (2007) 727 [math-ph/0610018] [INSPIRE].

[7] G. Slade, Critical Exponents for Long-Range $O(n)$ Models Below the Upper Critical Dimension, Commun. Math. Phys. 358 (2018) 343 [arXiv:1611.06169] [INSPIRE].

[8] P. Mitter, Long Range Ferromagnets: Renormalization Group Analysis, https://hal.archives-ouvertes.fr/cel-01239463.

[9] D. Benedetti, R. Gurau, S. Harribey and K. Suzuki, Long-range multi-scalar models at three loops, J. Phys. A 53 (2020) 445008 [arXiv:2007.04603] [InSPIRE].

[10] M.F. Paulos, S. Rychkov, B.C. van Rees and B. Zan, Conformal Invariance in the Long-Range Ising Model, Nucl. Phys. B 902 (2016) 246 [arXiv: 1509.00008] [INSPIRE].

[11] C. Behan, L. Rastelli, S. Rychkov and B. Zan, Long-range critical exponents near the short-range crossover, Phys. Rev. Lett. 118 (2017) 241601 [arXiv: 1703.03430] [INSPIRE].

[12] C. Behan, L. Rastelli, S. Rychkov and B. Zan, A scaling theory for the long-range to short-range crossover and an infrared duality, J. Phys. A 50 (2017) 354002 [arXiv: 1703.05325] [INSPIRE].

[13] C. Behan, Bootstrapping the long-range Ising model in three dimensions, J. Phys. A 52 (2019) 075401 [arXiv:1810.07199] [INSPIRE]. 
[14] J. Sak, Recursion Relations and Fixed Points for Ferromagnets with Long-Range Interactions, Phys. Rev. B 8 (1973) 281.

[15] J. Sak, Low-temperature renormalization group for ferromagnets with long-range interactions, Phys. Rev. B 15 (1977) 4344.

[16] E. Brezin, G. Parisi and F. Ricci-Tersenghi, The Crossover Region Between Long-Range and Short-Range Interactions for the Critical Exponents, J. Statist. Phys. 157 (2014) 855.

[17] S.S. Gubser, C. Jepsen, S. Parikh and B. Trundy, $O(N)$ and $O(N)$ and $O(N)$, JHEP 11 (2017) 107 [arXiv: 1703.04202] [INSPIRE].

[18] S. Giombi and H. Khanchandani, $O(N)$ models with boundary interactions and their long range generalizations, JHEP 08 (2020) 010 [arXiv: 1912.08169] [INSPIRE].

[19] M. Picco, Critical behavior of the Ising model with long range interactions, arXiv:1207.1018 [INSPIRE].

[20] T. Blanchard, M. Picco and M.A. Rajabpour, Influence of long-range interactions on the critical behavior of the Ising model, EPL 101 (2013) 56003 [arXiv:1211.6758] [INSPIRE].

[21] J. Honkonen and M.Y. Nalimov, Crossover between field theories with short range and long range exchange or correlations, J. Phys. A 22 (1989) 751 [InSPIRE].

[22] J. Honkonen, Critical behavior of the long range (phi**2)**2 model in the short range limit, J. Phys. A 23 (1990) 825 [inSPIRE].

[23] E. Luijten and H. Blote, Boundary between long-range and short-range critical behavior in systems with algebraic interactions, Phys. Rev. Lett. 89 (2002) 025703.

[24] L. Fei, S. Giombi and I.R. Klebanov, Critical $O(N)$ models in $6-\epsilon$ dimensions, Phys. Rev. D 90 (2014) 025018 [arXiv: 1404.1094] [INSPIRE].

[25] S.S. Gubser and I.R. Klebanov, A universal result on central charges in the presence of double trace deformations, Nucl. Phys. B 656 (2003) 23 [hep-th/0212138] [INSPIRE].

[26] M. Goykhman and M. Smolkin, Vector model in various dimensions, Phys. Rev. D 102 (2020) 025003 [arXiv:1911.08298] [INSPIRE].

[27] A.N. Vasiliev, Y.M. Pismak and Y.R. Khonkonen, Simple Method of Calculating the Critical Indices in the 1/N Expansion, Theor. Math. Phys. 46 (1981) 104 [InSPIRE].

[28] A.N. Vasiliev, Y.M. Pismak and Y.R. Khonkonen, 1/N Expansion: Calculation of the Exponents $\eta$ and $\nu$ in the Order $1 / N^{2}$ for Arbitrary Number of Dimensions, Theor. Math. Phys. 47 (1981) 465 [INSPIRE].

[29] S.E. Derkachov and A.N. Manashov, On the stability problem in the $O(N)$ nonlinear sigma model, Phys. Rev. Lett. 79 (1997) 1423 [hep-th/9705020] [INSPIRE].

[30] S.E. Derkachov and A.N. Manashov, Critical dimensions of composite operators in the nonlinear sigma model, Theor. Math. Phys. 116 (1998) 1034 [INSPIRE].

[31] S.-k. Ma, Scaling Variables and Dimensions, Phys. Rev. A 10 (1974) 1818 [InSPIRE].

[32] M. Goykhman, V. Rosenhaus and M. Smolkin, The background field method and critical vector models, JHEP 02 (2021) 074 [arXiv: 2009.13137] [INSPIRE].

[33] N. Chai, E. Rabinovici, R. Sinha and M. Smolkin, The bi-conical vector model at $1 / N$, JHEP 05 (2021) 192 [arXiv:2011.06003] [INSPIRE]. 
[34] N. Chai, M. Goykhman and R. Sinha, Conformal correlators in the critical $O(N)$ vector model, arXiv:2103.10168 [INSPIRE].

[35] A.M. Polyakov, Conformal symmetry of critical fluctuations, JETP Lett. 12 (1970) 381 [INSPIRE].

[36] A. Petkou, Conserved currents, consistency relations and operator product expansions in the conformally invariant $O(N)$ vector model, Annals Phys. 249 (1996) 180 [hep-th/9410093] [INSPIRE].

[37] M. Goykhman and R. Sinha, CFT data in the Gross-Neveu model, Phys. Rev. D 103 (2021) 125004 [arXiv: 2011.07768] [INSPIRE].

[38] S. Giombi and V. Kirilin, Anomalous dimensions in CFT with weakly broken higher spin symmetry, JHEP 11 (2016) 068 [arXiv:1601.01310] [INSPIRE].

[39] N. Chai, A. Dymarsky and M. Smolkin, A model of persistent breaking of discrete symmetry, arXiv:2106.09723 [INSPIRE].

[40] M. D'Eramo, G. Parisi and L. Peliti, Theoretical predictions for critical exponents at the lambda point of Bose liquids, Lett. Nuovo Cim. 2 (1971) 878 [INSPIRE].

[41] K. Symanzik, On calculations in conformal invariant field theories, Lett. Nuovo Cim. 3 (1972) 734 [INSPIRE]. 